\title{
The Neumann problem for a Barenblatt equation with a multiplicative stochastic force and a nonlinear source term
}

\author{
Caroline Bauzet, Frédéric Lebon and Asghar Maitlo
}

\begin{abstract}
In this paper, we are interested in an existence and uniqueness result for a Barenblatt's type equation forced by a multiplicative noise, with additionally a nonlinear source term and under Neumann boundary conditions. The idea to show such a well-posedness result is to investigate in a first step the additive case with a linear source term. Trough a time-discretization of the equation and thanks to results on maximal monotone operator, one is able to handle the non-linearity of the equation and pass to the limit on the discretization parameter. This allows us to show existence and uniqueness of a solution in the case of an additive noise and a linear source term. In a second step, thanks to a fixed point procedure, one shows the announced result.
\end{abstract}

Mathematics Subject Classification (2010). Primary 47J35, 60H15; Secondary 47H10, 47H05.

Keywords. Stochastic Barenblatt equation, multiplicative noise, additive noise, stochastic force, Itô integral, maximal monotone operator, Neumann condition, time discretization, heat equation, fixed point.

\section{Introduction}

We are concerned with the following stochastic PDEs of Barenblatt type involving respectively an additive noise:

$$
\left\{\begin{aligned}
\tilde{\alpha}\left(\partial_{t}\left(u-\int_{0} h d w\right)\right)-\Delta u & =g \text { in }(0, T) \times D \times \Omega, \\
\nabla u . \mathbf{n} & =0 \text { on }(0, T) \times \partial D \times \Omega, \\
u(0, .) & =u_{0}
\end{aligned}\right.
$$


and a multiplicative one with additionally a nonlinear source term:

$$
\left\{\begin{aligned}
\tilde{\alpha}\left(\partial_{t}\left(u-\int_{0} \mathscr{H}(u) d w\right)\right)-\Delta u+\beta(u) & =\vartheta \text { in }(0, T) \times D \times \Omega, \\
\nabla u . \mathbf{n} & =0 \text { on }(0, T) \times \partial D \times \Omega, \\
u(0, .) & =u_{0} .
\end{aligned}\right.
$$

We consider a standard adapted one-dimensional continuous Brownian motion

$$
w=\left\{w_{t}, \mathcal{F}_{t}, 0 \leqslant t \leqslant T\right\}
$$

defined on a complete probability space $(\Omega, \mathcal{F}, P)$ with a countably generated $\sigma$-field denoted $\mathcal{F}=\left(\mathcal{F}_{t}\right)_{t \geqslant 0}$ such that $w_{0}=0$ and $\mathcal{F}_{0}$ contains the negligible sets (see [11], [16] for further informations on stochastic analysis), the additive and multiplicative stochastic integrals $\int_{0} h d w$ and $\int_{0} \mathscr{H}(u) d w$ are understood in the sense of Itô, $D$ is a smooth bounded domain of $\mathbb{R}^{d}$ with $d \geqslant 1, \mathbf{n}$ is the outward normal vector to $\partial D$ and $u_{0}$ is a given initial condition. We assume the following hypotheses:

$H_{1}: h \in \mathcal{N}_{w}^{2}\left(0, T, H^{1}(D)\right) .^{\dagger}$

$H_{2}: \tilde{\alpha}=I_{d}+\alpha$ where $I_{d}: \mathbb{R} \rightarrow \mathbb{R}$ is the identity function and $\alpha: \mathbb{R} \rightarrow \mathbb{R}$ is a Lipschitz-continuous, coercive and non-decreasing function.

$H_{3}: g \in \mathcal{N}_{w}^{2}\left(0, T, L^{2}(D)\right)^{\dagger}$.

$H_{4}: u_{0} \in H^{1}(D)$.

$H_{5}: \mathscr{H}: \mathbb{R} \rightarrow \mathbb{R}$ is a Lipschitz-continuous function satisfying $\mathscr{H}(0)=0$.

$H_{6}: \beta: \mathbb{R} \rightarrow \mathbb{R}$ is a Lipschitz-continuous function.

$H_{7}: \vartheta \in \mathcal{N}_{w}^{2}\left(0, T, L^{2}(D)\right)^{\dagger}$.

In the deterministic case, these classes of Barenblatt equations (namely $f\left(\partial_{t} u\right)-\Delta u=g$, with $f$ a non-decreasing function) were originally considered by G.I. BARENBLATT in the theory of fluids in elasto-plastic porous medium [6]. Then, several studies around these equations were conducted in various areas: for applications in porous media models $[14,12,13]$, for irreversible phase change modeling [17] and for reaction-diffusion with absorption problems in Biochemistry [17]. More recently, the study of Barenblatt equations were revisited by different authors for constrained stratigraphic problems in Geology [1, 2, 3, 4, 19].

In the stochastic case, only few papers have been devoted to the study of Barenblatt equations with a stochastic force. Let us mention the work [5], where a related equation to the Barenblatt one with stochastic coefficients were studied. In [8], the authors were interested in abstract problem of Barenblatt's type with a stochastic force. Precisely, they investigated the Dirichlet problem for (1.1) in the case where the laplacian operator were replaced

\footnotetext{
${ }^{\dagger}$ For a given separable Hilbert space $X$, we denote by $\mathcal{N}_{w}^{2}(0, T, X)$ the space of predictable $X$-valued processes endowed with the norm $\|\phi\|_{\mathcal{N}_{w}^{2}(0, T, X)}^{2}=E\left[\int_{0}^{T}\|\phi\|_{X}^{2} d t\right]$ (see DA Prato-ZabCZyk [11] p.94).
} 
by a maximal monotone operator deriving from a potential. Then [7] proposed a result of existence and uniqueness of weak solution for equations (1.1) and (1.2) under Dirichlet boundary conditions and by assuming that $g=\beta=\vartheta=0$.

The aim of the present work is to complete the previous studies in the stochastic case by proposing an existence and uniqueness result of weak solution for a Barenblatt's type equation with a multiplicative noise $\int_{0}^{\cdot} \mathscr{H}(u) d w$, a non linear source term $\beta(u)$ and under Neumann boundary conditions. This paper represents an intermediate and preliminary work in view to aboard applications in phase transition phenomena (including irreversible phase changes such as solidification of glue, cooking an egg,...) trough the study of nonlinear evolution systems as in [9] with additionally a stochastic force.

For the sake of clarity, let us make precise some useful notations:

- $Q=(0, T) \times D$.

- $x . y$ the usual scalar product of $x$ and $y$ in $\mathbb{R}^{d}$.

- $\mathscr{D}(D)=\mathscr{C}_{c}^{\infty}(D)$ and $\mathscr{D}^{\prime}(D)$ the space of distributions on $D$.

- $\|$.$\| and (.,$.$) respectively the usual norm and the scalar product in L^{2}(D)$.

- $E[$.$] the expectation, i.e. the integral over \Omega$ with respect to the probability measure $P$.

- $C_{\alpha}>0$ the Lipschitz constant of $\alpha$.

- $\tilde{C}_{\alpha}>0$ the coerciveness constant of $\alpha$ : for any $x, y$ in $\mathbb{R}$,

$$
(\alpha(x)-\alpha(y))(x-y) \geqslant \tilde{C}_{\alpha}(x-y)^{2} .
$$

- $C_{\mathscr{H}}>0$ the Lipschitz constant of $\mathscr{H}$.

- $C_{\beta}>0$ the Lipschitz constant of $\beta$.

Now let us introduce the concept of solutions we are interested in for the two above problems and the main results of the paper.

Definition 1.1. Any predictable process $u$ belonging to $\mathcal{N}_{w}^{2}\left(0, T, H^{1}(D)\right)$, $L^{2}\left(\Omega, \mathscr{C}\left([0, T], L^{2}(D)\right)\right)$ and $\mathscr{C}\left([0, T], L^{2}\left(\Omega, H^{1}(D)\right)\right)$ is a solution to our stochastic problem (1.1) if $t$-almost everywhere in $(0, T), P$-almost surely in $\Omega$, the following variational formulation holds: for any $v \in H^{1}(D)$,

$$
\int_{D} \tilde{\alpha}\left(\partial_{t}\left(u-\int_{0}^{\cdot} h d w\right)\right) v d x+\int_{D} \nabla u . \nabla v d x=\int_{D} g v d x
$$

with $u(0,)=.u_{0} \in H^{1}(D)$.

Definition 1.2. Any predictable process $u$ belonging to $\mathcal{N}_{w}^{2}\left(0, T, H^{1}(D)\right)$, $L^{2}\left(\Omega, \mathscr{C}\left([0, T], L^{2}(D)\right)\right)$ and $\mathscr{C}\left([0, T], L^{2}\left(\Omega, H^{1}(D)\right)\right)$ is a solution to our stochastic problem (1.2) if $t$-almost everywhere in $(0, T), P$-almost surely in $\Omega$, 
the following variational formulation holds: for any $v \in H^{1}(D)$,

$$
\begin{aligned}
& \int_{D} \tilde{\alpha}\left(\partial_{t}\left(u-\int_{0} \mathscr{H}(u) d w\right)\right) v d x+\int_{D} \nabla u . \nabla v d x \\
& +\int_{D} \beta(u) v d x=\int_{D} \vartheta v d x,
\end{aligned}
$$

with $u(0,)=.u_{0} \in H^{1}(D)$.

Remark 1.3. Since the respective solutions of (1.1) and (1.2) belong to the set $L^{2}\left(\Omega, \mathscr{C}\left([0, T], L^{2}(D)\right)\right)$, they satisfy the initial condition in the following sense:

$$
\text { P-a.s, in } \Omega \quad u(t=0, .)=\lim _{t \rightarrow 0} u(t, .) \text { in } L^{2}(D) .
$$

The results we want to prove in the sequel are the following :

Theorem 1.4. Under assumptions $H_{1}$ to $H_{4}$, there exists a unique solution to Problem (1.1) in the sense of Definition 1.1.

Moreover, the solution of (1.1) depends continuously on the data, this is stated in the following proposition.

Proposition 1.5. Consider $g$, $\hat{g}$ in $\mathcal{N}_{w}^{2}\left(0, T, L^{2}(D)\right), h$, $\hat{h}$ in $\mathcal{N}_{w}^{2}\left(0, T, H^{1}(D)\right)$, $u_{0}, \hat{u}_{0}$ in $H^{1}(D)$ and denote by $u, \hat{u}$ the associated solutions to the Problem (1.1) in the sense of Definition 1.1 with the respective sets of data $\left(g, h, u_{0}\right)$ and $\left(\hat{g}, \hat{h}, \hat{u}_{0}\right)$. Then for any $t$ in $[0, T]$, by denoting $Q_{t}=(0, t) \times D$, the following inequality holds :

$$
\begin{aligned}
& \left(\tilde{C}_{\alpha}+\frac{1}{2}\right)\left\|\partial_{t}(U-\hat{U})\right\|_{L^{2}\left(\Omega \times Q_{t}\right)}^{2}+E\left[\|(u-\hat{u})(t)\|^{2}\right]+\frac{1}{2} E\left[\|\nabla(u-\hat{u})(t)\|^{2}\right] \\
\leqslant & C_{\alpha}^{T}\left\{\frac{1}{2}\left\|\nabla\left(u_{0}-\hat{u}_{0}\right)\right\|^{2}+\|\nabla(h-\hat{h})\|_{L^{2}\left(\Omega \times Q_{t}\right)}^{2}+\frac{1}{2}\|g-\hat{g}\|_{L^{2}\left(\Omega \times Q_{t}\right)}^{2}\right\}(1.6) \\
& +e^{t}\left\|u_{0}-\hat{u}_{0}\right\|^{2}+\|h-\hat{h}\|_{L^{2}\left(\Omega \times Q_{t}\right)}^{2},
\end{aligned}
$$

where $U=u-\int_{0}^{t} h d w, \hat{U}=\hat{u}-\int_{0}^{t} \hat{h} d w$ and $C_{\alpha}^{T}=1+e^{T}\left(\frac{2 C_{\alpha}^{2}}{\tilde{C}_{\alpha}+\frac{1}{2}}+T+4\right)$.

Theorem 1.6. Under Assumptions $\mathrm{H}_{2}$ to $\mathrm{H}_{7}$, there exists a unique solution to Problem (1.2) in the sense of Definition 1.2.

The paper is organized as follows. In a first step, we investigate the existence of a solution for Problem (1.1). The approach is the following: we use an implicit time discretization scheme to approximate such a solution. It relies on studying properties of the time-approximate solution and passing to the limit on the obtained discrete problem with respect to the discretization parameter. Because of the random variable, classical results of compactness do not hold, and the main difficulty here lies in the identification of the nonlinear term's limit associated with the discretization of $\alpha\left(\partial_{t}\left(u-\int_{0}^{\cdot} h d w\right)\right)$. In a second step, the uniqueness result is proven using classical energy estimates well known on the heat equation. In addition, one shows at the limit on the 
discretization parameter that the solution of Problem (1.1) depends continuously on the data (Proposition 1.5). By the way of a fixed-point theorem, we are able to extend in a last step our result of existence and uniqueness to the multiplicative case with additionally a nonlinear source term, that is the well-posedness of Problem (1.2).

\section{Study of the additive case}

The result of existence of a solution for Problem (1.1) is based on an implicit time discretization scheme for the deterministic part and an explicit one for the Itô part. To do so, let us introduce notations used for the discretization procedure.

\subsection{Notations and preliminaries results}

We consider $X$ a separable Banach space, $N \in \mathbb{N}^{*}$, set $\Delta t=\frac{T}{N}$ and $t_{n}=n \Delta t$ with $n \in\{0, \ldots, N\}$. For any sequence $\left(x_{n}\right)_{0 \leqslant n \leqslant N} \subset X$, let us denote by

$$
\begin{aligned}
x^{\Delta t} & =\sum_{k=0}^{N-1} x_{k+1} \mathbb{1}_{\left[t_{k}, t_{k+1}\right)}, \\
x_{\Delta t} & =\sum_{k=0}^{N-1} x_{k} \mathbb{1}_{\left[t_{k}, t_{k+1}\right)}=x^{\Delta t}(.-\Delta t), \\
\tilde{x}^{\Delta t} & =\sum_{k=0}^{N-1}\left[\frac{x_{k+1}-x_{k}}{\Delta t}\left(.-t_{k}\right)+x_{k}\right] \mathbb{1}_{\left[t_{k}, t_{k+1}\right)}, \\
\frac{\partial \tilde{x}^{\Delta t}}{\partial t} & =\sum_{k=0}^{N-1} \frac{x_{k+1}-x_{k}}{\Delta t} \mathbb{1}_{\left[t_{k}, t_{k+1}\right)},
\end{aligned}
$$

with the convention that $t_{-1}=-\Delta t$, for $t<0, \tilde{x}^{\Delta t}(t)=x_{0}$ and $x^{\Delta t}\left(t_{N}\right)=$ $\tilde{x}^{\Delta t}\left(t_{N}\right)=x_{N}$. Elementary calculations yield for an arbitrary constant $C>0$ independent of $\Delta t$

$$
\begin{aligned}
& \left\|x^{\Delta t}\right\|_{L^{2}(0, T ; X)}^{2}=\Delta t \sum_{k=1}^{N}\left\|x_{k}\right\|_{X}^{2} ; \quad\left\|\tilde{x}^{\Delta t}\right\|_{L^{2}(0, T ; X)}^{2} \leqslant C \Delta t \sum_{k=0}^{N}\left\|x_{k}\right\|_{X}^{2} ; \\
& \left\|x^{\Delta t}-\tilde{x}^{\Delta t}\right\|_{L^{2}(0, T ; X)}^{2}=\Delta t \sum_{k=0}^{N-1}\left\|x_{k+1}-x_{k}\right\|_{X}^{2} ; \\
& \left\|x^{\Delta t}(.-\Delta t)-x^{\Delta t}\right\|_{L^{2}(0, T ; X)}^{2}=\Delta t \sum_{k=0}^{N-1}\left\|x_{k+1}-x_{k}\right\|_{X}^{2} ; \\
& \left\|\frac{\partial \tilde{x}^{\Delta t}}{\partial t}\right\|_{L^{2}(0, T ; X)}^{2}=\frac{1}{\Delta t} \sum_{k=0}^{N-1}\left\|x_{k+1}-x_{k}\right\|_{X}^{2} ; \\
& \left\|x^{\Delta t}\right\|_{L^{\infty}(0, T ; X)}=\max _{k=1, . ., N}\left\|x_{k}\right\|_{X} \text { and }\left\|\tilde{x}^{\Delta t}\right\|_{L^{\infty}(0, T ; X)}=\max _{k=0, . ., N}\left\|x_{k}\right\|_{X} .
\end{aligned}
$$


We will use the following notations for the discretization of the data for any $n$ in $\{0, \ldots, N\}$ :

$$
\begin{aligned}
& w_{n}=w\left(t_{n}\right), h_{n}=\frac{1}{\Delta t} \int_{t_{n-1}}^{t_{n}} h(s, .) d s, B_{n}=\sum_{k=0}^{n-1}\left(w_{k+1}-w_{k}\right) h_{k} \\
& \text { and } g_{n}=\frac{1}{\Delta t} \int_{t_{n-1}}^{t_{n}} g(s, .) d s,
\end{aligned}
$$

with the convention that $t_{-1}=-\Delta t$ and $h(s,)=.g(s,)=$.0 if $s<0$.

Remark 2.1. As $h$ and $g$ are predictable, $h_{n}$ and $g_{n}$ belong respectively to $L^{2}\left(\left(\Omega, \mathcal{F}_{t_{n}}\right) ; H^{1}(D)\right)$ and $L^{2}\left(\left(\Omega, \mathcal{F}_{t_{n}}\right) ; L^{2}(D)\right)$ for any $n$ in $\{0, \ldots, N\}$.

Remark 2.2. For any $n$ in $\{0, \ldots, N\}, B_{n}=\int_{0}^{t_{n}} h_{\Delta t}(s) d w(s)$.

Indeed, as $h_{k}$ is $\mathcal{F}_{t_{k}}$-measurable, one has

$$
\begin{aligned}
B_{n} & =\sum_{k=0}^{n-1}\left(w_{k+1}-w_{k}\right) h_{k} \\
& =\sum_{k=0}^{n-1} \int_{t_{k}}^{t_{k+1}} h_{k} d w(s) \\
& =\int_{0}^{t_{n}} \sum_{k=0}^{n-1} h_{k} \mathbb{1}_{\left[t_{k}, t_{k+1}[\right.}(s) d w(s) \\
& =\int_{0}^{t_{n}} h_{\Delta t}(s) d w(s) .
\end{aligned}
$$

Lemma 2.3. There exists a constant $C \geqslant 0$ independent of $\Delta t$ such that for any $n$ in $\{0, \ldots, N\}$

$$
E\left[\sum_{k=0}^{n}\left\|h_{k}\right\|_{H^{1}(D)}^{2}\right] \leqslant \frac{C}{\Delta t} \quad \text { and } \quad E\left[\sum_{k=0}^{n}\left\|g_{k}\right\|^{2}\right] \leqslant \frac{C}{\Delta t} .
$$

Proof. Since for any $n \in\{0, \ldots, N\}$ and any $k \in\{0, \ldots, n\}$

$$
\begin{aligned}
\left\|h_{k}\right\|_{H^{1}(D)}^{2} & =\left\|\frac{1}{\Delta t} \int_{t_{k-1}}^{t_{k}} h(s) d s\right\|_{H^{1}(D)}^{2} \\
& \leqslant \frac{1}{\Delta t^{2}}\left(\int_{t_{k-1}}^{t_{k}}\|h(s)\|_{H^{1}(D)} d s\right)^{2} \\
& \leqslant \frac{1}{\Delta t^{2}} \times \Delta t \int_{t_{k-1}}^{t_{k}}\|h(s)\|_{H^{1}(D)}^{2} d s
\end{aligned}
$$

one gets :

$$
\begin{aligned}
E\left[\sum_{k=0}^{n}\left\|h_{k}\right\|_{H^{1}(D)}^{2}\right] & \leqslant \frac{1}{\Delta t} E\left[\sum_{k=0}^{n} \int_{t_{k-1}}^{t_{k}}\|h(s)\|_{H^{1}(D)}^{2} d s\right] \\
& \leqslant \frac{1}{\Delta t}\|h\|_{L^{2}\left((0, T) \times \Omega, H^{1}(D)\right)}^{2} \leqslant \frac{C}{\Delta t} .
\end{aligned}
$$


The proof of the second estimate is the same.

Lemma 2.4. The sequences $\left(h_{\Delta t}\right)$ and $\left(g_{\Delta t}\right)$ converge to $h$ and $g$ respectively in $\mathcal{N}_{w}^{2}\left(0, T, H^{1}(D)\right)$ and $\mathcal{N}_{w}^{2}\left(0, T, L^{2}(D)\right)$ as the time discretization parameter $\Delta t$ tends to 0 .

Proof. See Simon [18], Lemma 12 p.52.

Proposition 2.5. The sequences $\left(B^{\Delta t}\right)$ and $\left(\tilde{B}^{\Delta t}\right)$ converge to $\int_{0}^{\cdot} h d w$ in $L^{2}\left(0, T ; L^{2}\left(\Omega, H^{1}(D)\right)\right)$ as the time discretization parameter $\Delta t$ tends to 0 .

Proof. Using successively Lemma 2.3, Itô isometry and Lemma 2.4, we have

$$
\begin{aligned}
& E\left[\int_{0}^{T}\left\|\tilde{B}^{\Delta t}-\int_{0}^{\cdot} h d w\right\|_{H^{1}(D)}^{2} d s\right] \\
= & E\left[\sum_{k=0}^{n-1} \int_{t_{k}}^{t_{k+1}}\left\|\frac{\left(w_{k+1}-w_{k}\right)}{\Delta t} h_{k}\left(s-t_{k}\right)+B_{k}-\int_{0}^{s} h(\sigma) d w(\sigma)\right\|_{H^{1}(D)}^{2} d s\right] \\
\leqslant & 2 E\left[\sum_{k=0}^{n-1} \int_{t_{k}}^{t_{k+1}}\left|\frac{w_{k+1}-w_{k}}{\Delta t}\right|^{2}\left(s-t_{k}\right)^{2}\left\|h_{k}\right\|_{H^{1}(D)}^{2} d s\right] \\
& +2 E\left[\sum_{k=0}^{n-1} \int_{t_{k}}^{t_{k+1}}\left\|B_{k}-\int_{0}^{s} h(\sigma) d w(\sigma)\right\|_{H^{1}(D)}^{2} d s\right] \\
\leqslant & C \Delta t^{2} E\left[\sum_{k=0}^{n-1}\left\|h_{k}\right\|^{2}\right]+C E\left[\sum_{k=0}^{n-1} \int_{t_{k}}^{t_{k+1}}\left\|\int_{t_{k}}^{s} h(\sigma) d w(\sigma)\right\|_{H^{1}(D)}^{2} d s\right] \\
& +C E\left[\sum_{k=0}^{n-1} \int_{t_{k}}^{t_{k+1}}\left\|\int_{0}^{t_{k}}\left(h_{\Delta t}-h\right)(\sigma) d w(\sigma)\right\|_{H^{1}(D)}^{2} d s\right] \\
\leqslant & C \Delta t+C E\left[\sum_{k=0}^{n-1} \int_{t_{k}}^{t_{k+1}} \int_{0}^{t_{k}}\left\|\left(h_{\Delta t}-h\right)(\sigma)\right\|_{H^{1}(D)}^{2} d \sigma d s\right] \\
\leqslant & \left.C \Delta t+C\left\|h_{\Delta t}-h\right\|_{L^{2}\left((0, T) \times \Omega, H^{1}(D)\right)}^{2}+C \Delta t\|h\|_{L^{2}\left((0, T) \times \Omega, H^{1}(D)\right)}^{n-1} \int_{t_{k}}^{t_{k+1}} \int_{t_{k}}^{s}\|h(\sigma)\|_{H^{1}(D)}^{2} d \sigma d s\right] \\
& +C E[
\end{aligned}
$$

and so

$$
\tilde{B}^{\Delta t} \rightarrow \int_{0}^{\cdot} h d w \text { in } L^{2}\left((0, T) \times \Omega, H^{1}(D)\right) \text { as } \Delta t \rightarrow 0
$$


Moreover, thanks to Lemma 2.3

$$
\begin{aligned}
\left\|B^{\Delta t}-\tilde{B}^{\Delta t}\right\|_{L^{2}\left((0, T) \times \Omega, H^{1}(D)\right)}^{2} & =\Delta t E\left[\sum_{k=0}^{N-1}\left\|B_{k+1}-B_{k}\right\|_{H^{1}(D)}^{2}\right] \\
& =\Delta t E\left[\sum_{k=0}^{N-1}\left\|\left(w_{k+1}-w_{k}\right) h_{k}\right\|_{H^{1}(D)}^{2}\right] \\
& =\Delta t^{2} E\left[\sum_{k=0}^{N-1}\left\|h_{k}\right\|_{H^{1}(D)}^{2}\right] \\
& \leqslant C \Delta t,
\end{aligned}
$$

and so $B^{\Delta t} \rightarrow \int_{0}^{\cdot} h d w$ in $L^{2}\left((0, T) \times \Omega, H^{1}(D)\right)$ as $\Delta t \rightarrow 0$.

Remark 2.6. If one assumes that $h$ belongs to $\mathcal{N}_{w}^{2}\left(0, T, H^{2}(D)\right)$, one shows in the same manner that $B^{\Delta t}$ converges strongly to $\int_{0}^{\cdot} h d w$ in $L^{2}((0, T) \times$ $\left.\Omega, H^{2}(D)\right)$ as $\Delta t$ tends to 0 .

\subsection{Discretization scheme}

We consider a positive integer $N$ and $n \in\{0, \ldots, N\}$. Using the notations of the previous section, the discretization scheme is the following one: for given small positive parameter $\Delta t$ and $u_{n}$ in $L^{2}\left(\left(\Omega, \mathcal{F}_{t_{n}}\right) ; H^{1}(D)\right)$, we aim to find $u_{n+1}$ in $L^{2}\left(\left(\Omega, \mathcal{F}_{t_{n+1}}\right) ; H^{1}(D)\right)$, such that $P$-a.s in $\Omega$ and for any $v$ in $H^{1}(D)$

$$
\begin{aligned}
& \int_{D}\left(\frac{u_{n+1}-u_{n}}{\Delta t}-h_{n} \frac{w_{n+1}-w_{n}}{\Delta t}\right) v d x+\int_{D} \nabla u_{n+1} \cdot \nabla v d x \\
& +\int_{D} \alpha\left(\frac{u_{n+1}-u_{n}}{\Delta t}-h_{n} \frac{w_{n+1}-w_{n}}{\Delta t}\right) v d x=\int_{D} g_{n} v d x .
\end{aligned}
$$

To proceed in this way we prove that, once $n$ is fixed, we can find the solution for the step $n+1$ by a fixed point argument.

Proposition 2.7. Set $N \in \mathbb{N}^{*}, n \in\{0, \ldots, N\}$ and $u_{n} \in L^{2}\left(\left(\Omega, \mathcal{F}_{t_{n}}\right) ; H^{1}(D)\right)$. Then there exists a unique $u_{n+1} \in L^{2}\left(\left(\Omega, \mathcal{F}_{t_{n+1}}\right) ; H^{1}(D)\right)$ such that $P$-a.s in $\Omega$ and for any $v$ in $H^{1}(D)$

$$
\begin{aligned}
& \int_{D}\left(\frac{u_{n+1}-u_{n}}{\Delta t}-h_{n} \frac{w_{n+1}-w_{n}}{\Delta t}\right) v d x+\int_{D} \nabla u_{n+1} \cdot \nabla v d x \\
& +\int_{D} \alpha\left(\frac{u_{n+1}-u_{n}}{\Delta t}-h_{n} \frac{w_{n+1}-w_{n}}{\Delta t}\right) v d x=\int_{D} g_{n} v d x .
\end{aligned}
$$

Proof. Set $N \in \mathbb{N}^{*}, n \in\{0, \ldots, N\}$ and $u_{n} \in L^{2}\left(\left(\Omega, \mathcal{F}_{t_{n}}\right) ; H^{1}(D)\right)$. The variational problem $(2.1)$ can be rewritten in the following way : finding $U_{n+1} \in L^{2}\left(\left(\Omega, \mathcal{F}_{t_{n+1}}\right) ; H^{1}(D)\right)$ such that $P$-a.s in $\Omega$ and for any $v$ in $H^{1}(D)$

$$
\begin{aligned}
& \int_{D} U_{n+1} v d x+\Delta t \int_{D} \nabla U_{n+1} \cdot \nabla v d x+\int_{D} \alpha\left(U_{n+1}\right) v d x \\
& =\int_{D} g_{n} v d x-\int_{D} \nabla\left(u_{n}+h_{n}\left(w_{n+1}-w_{n}\right)\right) \cdot \nabla v d x .
\end{aligned}
$$


The idea to solve (2.2) is to use the fixed-point theorem of Banach on the following equivalent problem for a fixed $\lambda>0$

$$
\begin{aligned}
& (\lambda+1) \int_{D} U_{n+1} v d x+\lambda \Delta t \int_{D} \nabla U_{n+1} \cdot \nabla v d x=\int_{D}\left(U_{n+1}-\lambda \alpha\left(U_{n+1}\right)\right) v d x \\
& +\lambda \int_{D} g_{n} v d x-\lambda \int_{D} \nabla\left(u_{n}+h_{n}\left(w_{n+1}-w_{n}\right)\right) . \nabla v d x
\end{aligned}
$$

To do so, we introduce the following application, for $\lambda>0$

$$
\begin{aligned}
J_{\lambda}: L^{2}\left(\Omega ; L^{2}(D)\right) & \rightarrow L^{2}\left(\Omega ; L^{2}(D)\right) \\
v & \mapsto v-\lambda \alpha(v) .
\end{aligned}
$$

Firstly, thanks to the monotonicity of $\alpha$, one shows that under the condition $0<\lambda \leqslant \frac{1}{C_{\alpha}}, J_{\lambda}$ is a contraction. Secondly, one considers for $0<\lambda \leqslant \frac{1}{C_{\alpha}}$ the application

$$
\begin{aligned}
T_{\lambda}: L^{2}\left(\left(\Omega, \mathcal{F}_{t_{n+1}}\right) ; L^{2}(D)\right) & \rightarrow L^{2}\left(\left(\Omega, \mathcal{F}_{t_{n+1}}\right) ; H^{1}(D)\right) \\
S & \mapsto u_{S}
\end{aligned}
$$

where $u_{S}$ is the solution in $L^{2}\left(\left(\Omega, \mathcal{F}_{t_{n+1}}\right) ; H^{1}(D)\right)$ of the following variational problem, $P$-a.s in $\Omega$ and for any $v$ in $H^{1}(D)$

$$
\begin{aligned}
& (\lambda+1) \int_{D} u_{S} v d x+\lambda \Delta t \int_{D} \nabla u_{S} \cdot \nabla v d x=\int_{D}(S-\lambda \alpha(S)) v d x \\
& +\lambda \int_{D} g_{n} v d x-\lambda \int_{D} \nabla\left(u_{n}+h_{n}\left(w_{n+1}-w_{n}\right)\right) \cdot \nabla v d x .
\end{aligned}
$$

Note that thanks to the Lax-Milgram theorem, $T_{\lambda}$ is well defined. Using the contraction property of $J_{\lambda}$, one shows that $T_{\lambda}$ is a strict contraction in $L^{2}\left(\left(\Omega, \mathcal{F}_{t_{n+1}}\right) ; L^{2}(D)\right)$, thus using the Banach fixed-point theorem, one gets the existence and uniqueness of a solution for (2.3) denoted $U_{n+1}$ in $L^{2}\left(\left(\Omega, \mathcal{F}_{t_{n+1}}\right) ; H^{1}(D)\right)$. By setting $u_{n+1}=\Delta t U_{n+1}+u_{n}+h_{n}\left(w_{n+1}-w_{n}\right)$, one gets that $(2.1)$ admits a unique solution in $L^{2}\left(\left(\Omega, \mathcal{F}_{t_{n+1}}\right) ; H^{1}(D)\right)$.

\subsection{Estimates on the approximate sequence}

We propose the following discretization of our variational formulation (1.4) : t-almost everywhere in $(0, T), P$-almost surely in $\Omega$ and for any $v$ in $H^{1}(D)$

$$
\int_{D} \tilde{\alpha}\left(\partial_{t}\left(\tilde{u}^{\Delta t}-\tilde{B}^{\Delta t}\right)\right) v d x+\int_{D} \nabla u^{\Delta t} \cdot \nabla v d x=\int_{D} g_{\Delta t} v d x
$$

The aim here is to obtain boundedness results for the sequences $\tilde{u}^{\Delta t}, u^{\Delta t}$ and $\tilde{u}^{\Delta t}-\tilde{B}^{\Delta t}$. 
Proposition 2.8. A constant $C>0$ independent of $\Delta t$ exists such that

$$
\begin{aligned}
\left\|\nabla \tilde{u}^{\Delta t}\right\|_{L^{\infty}\left(0, T, L^{2}(\Omega \times D)\right)},\left\|\nabla u^{\Delta t}\right\|_{L^{\infty}\left(0, T ; L^{2}(\Omega \times D)\right)} & \leq C, \\
\left\|\tilde{u}^{\Delta t}-u^{\Delta t}\right\|_{L^{2}\left((0, T) \times \Omega, H^{1}(D)\right)} & \leq C \sqrt{\Delta t}, \\
\left\|\tilde{u}^{\Delta t}-\tilde{u}^{\Delta t}(.-\Delta t)\right\|_{L^{2}\left((0, T) \times \Omega, H^{1}(D)\right)} & \leq C \sqrt{\Delta t}, \\
\left\|\partial_{t}\left(\tilde{u}^{\Delta t}-\tilde{B}^{\Delta t}\right)\right\|_{L^{2}(\Omega \times Q)} & \leq C, \\
\left\|\tilde{u}^{\Delta t}-\tilde{B}^{\Delta t}\right\|_{L^{\infty}\left(0, T ; L^{2}(\Omega \times D)\right)} & \leq C, \\
\left\|\nabla\left(\tilde{u}^{\Delta t}-\tilde{B}^{\Delta t}\right)\right\|_{L^{2}(\Omega \times Q)} & \leq C, \\
\left\|\tilde{u}^{\Delta t}\right\|_{L^{2}\left((0, T) \times \Omega, H^{1}(D)\right)},\left\|u^{\Delta t}\right\|_{L^{2}\left((0, T) \times \Omega, H^{1}(D)\right)} & \leq C, \\
\left\|\tilde{u}^{\Delta t}(.-\Delta t)\right\|_{\mathcal{N}_{w}^{2}\left(0, T, H^{1}(D)\right)} & \leq C .
\end{aligned}
$$

Proof. Set $N \in \mathbb{N}^{*}, n \in\{0, . ., N-1\}$ and $k \in\{0, \ldots, n\}$. The variational formulation (2.1) with the couple of indexes $(k+1, k)$ and the particular test function $v=\frac{u_{k+1}-u_{k}}{\Delta t}-h_{k} \frac{w_{k+1}-w_{k}}{\Delta t}$ gives us

$$
\begin{aligned}
& \left\|\frac{u_{k+1}-u_{k}}{\Delta t}-h_{k} \frac{w_{k+1}-w_{k}}{\Delta t}\right\|^{2} \\
& +\int_{D} \alpha\left(\frac{u_{k+1}-u_{k}}{\Delta t}-h_{k} \frac{w_{k+1}-w_{k}}{\Delta t}\right)\left(\frac{u_{k+1}-u_{k}}{\Delta t}-h_{k} \frac{w_{k+1}-w_{k}}{\Delta t}\right) d x \\
& +\int_{D} \nabla u_{k+1} \cdot \nabla\left(\frac{u_{k+1}-u_{k}}{\Delta t}-h_{k} \frac{w_{k+1}-w_{k}}{\Delta t}\right) d x=\int_{D} g_{k} v d x
\end{aligned}
$$

Then

$$
\begin{aligned}
& \left(\tilde{C}_{\alpha}+1\right)\left\|\frac{u_{k+1}-u_{k}}{\Delta t}-h_{k} \frac{w_{k+1}-w_{k}}{\Delta t}\right\|^{2} \\
& +\int_{D} \nabla u_{k+1} \cdot \nabla\left(\frac{u_{k+1}-u_{k}}{\Delta t}-h_{k} \frac{w_{k+1}-w_{k}}{\Delta t}\right) d x \leq \int_{D} g_{k} v d x .
\end{aligned}
$$

Moreover for any $\delta>0$,

$$
\begin{aligned}
& \left(\tilde{C}_{\alpha}+1\right)\left\|\frac{u_{k+1}-u_{k}}{\Delta t}-h_{k} \frac{w_{k+1}-w_{k}}{\Delta t}\right\|^{2}+\int_{D} \nabla u_{k+1} \cdot \nabla \frac{u_{k+1}-u_{k}}{\Delta t} d x \\
& -\int_{D} \nabla\left(u_{k+1}-u_{k}\right) \cdot \nabla h_{k} \frac{w_{k+1}-w_{k}}{\Delta t} d x-\int_{D} \nabla u_{k} \cdot \nabla h_{k} \frac{w_{k+1}-w_{k}}{\Delta t} d x \\
\leq & \frac{\delta}{2}\left\|\frac{u_{k+1}-u_{k}}{\Delta t}-h_{k} \frac{w_{k+1}-w_{k}}{\Delta t}\right\|^{2}+\frac{1}{2 \delta}\left\|g_{k}\right\|^{2},
\end{aligned}
$$

and

$$
\int_{D} \nabla u_{k+1} \cdot \nabla \frac{u_{k+1}-u_{k}}{\Delta t} d x=\frac{1}{2 \Delta t}\left[\left\|\nabla u_{k+1}\right\|^{2}-\left\|\nabla u_{k}\right\|^{2}+\left\|\nabla\left(u_{k+1}-u_{k}\right)\right\|^{2}\right] .
$$


Using this with $\delta=\tilde{C}_{\alpha}+1$ and for all $\epsilon>0$, one gets

$$
\begin{aligned}
& \frac{\tilde{C}_{\alpha}+1}{2}\left\|\frac{u_{k+1}-u_{k}}{\Delta t}-h_{k} \frac{w_{k+1}-w_{k}}{\Delta t}\right\|^{2} \\
& +\frac{1}{2 \Delta t}\left[\left\|\nabla u_{k+1}\right\|^{2}-\left\|\nabla u_{k}\right\|^{2}+\left\|\nabla\left(u_{k+1}-u_{k}\right)\right\|^{2}\right] \\
\leq & \frac{\epsilon}{2 \Delta t}\left\|\nabla\left(u_{k+1}-u_{k}\right)\right\|^{2}+\frac{\left|w_{k+1}-w_{k}\right|^{2}}{2 \epsilon \Delta t}\left\|\nabla h_{k}\right\|^{2} \\
& -\int_{D} \nabla u_{k} \cdot \nabla h_{k} \frac{w_{k+1}-w_{k}}{\Delta t} d x+\frac{1}{2\left(\tilde{C}_{\alpha}+1\right)}\left\|g_{k}\right\|^{2} .
\end{aligned}
$$

Then, since $u_{k}$ and $h_{k}$ are $\mathcal{F}_{t_{k}}$-measurable, by taking the expectation one gets

$$
\begin{aligned}
& \left(\tilde{C}_{\alpha}+1\right) E\left[\left\|\frac{u_{k+1}-u_{k}}{\Delta t}-h_{k} \frac{w_{k+1}-w_{k}}{\Delta t}\right\|^{2}\right] \\
& +\frac{1}{\Delta t} E\left[\left\|\nabla u_{k+1}\right\|^{2}-\left\|\nabla u_{k}\right\|^{2}+\left\|\nabla\left(u_{k+1}-u_{k}\right)\right\|^{2}\right] \\
\leq & \frac{\epsilon}{\Delta t} E\left[\left\|\nabla\left(u_{k+1}-u_{k}\right)\right\|^{2}\right]+\frac{1}{\epsilon} E\left[\left\|\nabla h_{k}\right\|^{2}\right]+\frac{1}{\tilde{C}_{\alpha}+1} E\left[\left\|g_{k}\right\|^{2}\right] .
\end{aligned}
$$

In this way

$$
\begin{aligned}
& \left(\tilde{C}_{\alpha}+1\right) \Delta t E\left[\left\|\frac{u_{k+1}-u_{k}}{\Delta t}-h_{k} \frac{w_{k+1}-w_{k}}{\Delta t}\right\|^{2}\right] \\
& +E\left[\left\|\nabla u_{k+1}\right\|^{2}-\left\|\nabla u_{k}\right\|^{2}\right]+(1-\epsilon) E\left[\left\|\nabla\left(u_{k+1}-u_{k}\right)\right\|^{2}\right] \\
\leq & \frac{\Delta t}{\epsilon} E\left[\left\|\nabla h_{k}\right\|^{2}\right]+\frac{\Delta t}{\tilde{C}_{\alpha}+1} E\left[\left\|g_{k}\right\|^{2}\right],
\end{aligned}
$$

and by summing from $k=0$ to $n$, one gets

$$
\begin{aligned}
& \left(\tilde{C}_{\alpha}+1\right) \sum_{k=0}^{n} \Delta t E\left[\left\|\frac{u_{k+1}-u_{k}}{\Delta t}-\frac{w_{k+1}-w_{k}}{\Delta t} h_{k}\right\|^{2}\right] \\
& +\sum_{k=0}^{n} E\left[\left\|\nabla u_{k+1}\right\|^{2}-\left\|\nabla u_{k}\right\|^{2}\right]+(1-\epsilon) \sum_{k=0}^{n} E\left[\left\|\nabla\left(u_{k+1}-u_{k}\right)\right\|^{2}\right] \\
\leq & \frac{1}{\epsilon} \sum_{k=0}^{n} \Delta t E\left[\left\|\nabla h_{k}\right\|^{2}\right]+\frac{1}{\tilde{C}_{\alpha}+1} \sum_{k=0}^{n} \Delta t E\left[\left\|g_{k}\right\|^{2}\right]
\end{aligned}
$$


and thanks to Lemma 2.3, there exists a constant $C$ independent of $\Delta t$ such that

$$
\begin{aligned}
& \left(\tilde{C}_{\alpha}+1\right) \sum_{k=0}^{n} \Delta t E\left[\left\|\frac{u_{k+1}-u_{k}}{\Delta t}-h_{k} \frac{w_{k+1}-w_{k}}{\Delta t}\right\|^{2}\right] \\
& +E\left[\left\|\nabla u_{n+1}\right\|^{2}\right]+(1-\epsilon) \sum_{k=0}^{n} E\left[\left\|\nabla\left(u_{k+1}-u_{k}\right)\right\|^{2}\right] \\
& \leq \frac{1}{\epsilon} \sum_{k=0}^{n} \Delta t E\left[\left\|\nabla h_{k}\right\|^{2}\right]+\frac{1}{\tilde{C}_{\alpha}+1} \sum_{k=0}^{n} \Delta t E\left[\left\|g_{k}\right\|^{2}\right]+\left\|\nabla u_{0}\right\|^{2} \\
& \leq C .
\end{aligned}
$$

Consequently, by taking $\epsilon=1 / 2$ for example, we obtain

$$
\begin{aligned}
& \sum_{k=0}^{n} \Delta t E\left[\left\|\frac{u_{k+1}-u_{k}}{\Delta t}-h_{k} \frac{w_{k+1}-w_{k}}{\Delta t}\right\|^{2}\right]+E\left[\left\|\nabla u_{n+1}\right\|^{2}\right] \\
& +\frac{1}{2} \sum_{k=0}^{n} E\left\|\nabla\left(u_{k+1}-u_{k}\right)\right\|^{2} \leq C,
\end{aligned}
$$

and we get directly the following estimates:

$$
\begin{aligned}
\left\|\nabla \tilde{u}^{\Delta t}\right\|_{L^{\infty}\left(0, T, L^{2}(\Omega \times D)\right)},\left\|\nabla u^{\Delta t}\right\|_{L^{\infty}\left(0, T, L^{2}(\Omega \times D)\right)} & \leq C, \\
\left\|\nabla\left(\tilde{u}^{\Delta t}-u^{\Delta t}\right)\right\|_{L^{2}(\Omega \times Q)} & \leq C \sqrt{\Delta t}, \\
\left\|\partial_{t}\left(\tilde{u}^{\Delta t}-\tilde{B}^{\Delta t}\right)\right\|_{L^{2}(\Omega \times Q)} & \leq C .
\end{aligned}
$$

Using (2.13), we have

$$
\begin{aligned}
& \left\|\tilde{u}^{\Delta t}-u^{\Delta t}\right\|_{L^{2}(\Omega \times Q)}^{2}=\Delta t \sum_{k=0}^{N-1} E\left[\left\|u_{k+1}-u_{k}\right\|^{2}\right] \\
\leqslant & \Delta t \sum_{k=0}^{N-1} E\left[2 \Delta t^{2}\left\|\frac{u_{k+1}-u_{k}}{\Delta t}-h_{k} \frac{w_{k+1}-w_{k}}{\Delta t}\right\|^{2}+2\left\|h_{k}\left(w_{k+1}-w_{k}\right)\right\|^{2}\right] \\
= & 2 \Delta t^{2} \sum_{k=0}^{N-1} \Delta t E\left[\left\|\frac{u_{k+1}-u_{k}}{\Delta t}-h_{k} \frac{w_{k+1}-w_{k}}{\Delta t}\right\|^{2}\right]+2 \sum_{k=0}^{N-1} \Delta t^{2} E\left[\left\|h_{k}\right\|^{2}\right] \\
\leqslant & C \Delta t .
\end{aligned}
$$

In the same manner, since

$$
\left\|u^{\Delta t}-u^{\Delta t}(.-\Delta t)\right\|_{L^{2}(\Omega \times Q)}^{2}=\Delta t \sum_{k=0}^{N-1} E\left[\left\|u_{k+1}-u_{k}\right\|^{2}\right],
$$

one gets also that

$$
\left\|u^{\Delta t}-u^{\Delta t}(.-\Delta t)\right\|_{L^{2}(\Omega \times Q)}^{2} \leq C \Delta t .
$$


Now, using the equality

$$
\left\|\nabla\left(u^{\Delta t}-u^{\Delta t}(.-\Delta t)\right)\right\|_{L^{2}(\Omega \times Q)}^{2}=\Delta t \sum_{k=0}^{N-1} E\left[\left\|\nabla\left(u_{k+1}-u_{k}\right)\right\|^{2}\right]
$$

one obtains due to (2.13) that

$$
\left\|\nabla\left(u^{\Delta t}-u^{\Delta t}(.-\Delta t)\right)\right\|_{L^{2}(\Omega \times Q)}^{2} \leq C \Delta t .
$$

Similarly, one shows that

$$
\begin{aligned}
& \left\|u^{\Delta t}(.-\Delta t)-\tilde{u}^{\Delta t}(.-\Delta t)\right\|_{L^{2}\left((0, T) \times \Omega, H^{1}(D)\right)}^{2} \\
\leqslant & C \sum_{k=0}^{N-1} \Delta t E\left[\left\|u_{k+1}-u_{k}\right\|_{H^{1}(D)}^{2}\right] \\
\leqslant & C \Delta t
\end{aligned}
$$

and by combining this with the previous inequalities one gets that

$$
\left\|\tilde{u}^{\Delta t}-\tilde{u}^{\Delta t}(.-\Delta t)\right\|_{L^{2}\left((0, T) \times \Omega, H^{1}(D)\right)} \leqslant C \sqrt{\Delta t} .
$$

Additionally for any $n$ in $\{0, \ldots, N-1\}$ since $B_{0}=0$ one has

$$
\begin{aligned}
& E\left[\left\|u_{n+1}-B_{n+1}\right\|^{2}\right] \\
= & E\left[\left\|u_{0}+\sum_{k=0}^{n} u_{k+1}-u_{k}-\left(B_{k+1}-B_{k}\right)\right\|^{2}\right] \\
\leqslant & 2\left\|u_{0}\right\|^{2}+2 \Delta t^{2} E\left[\left\|\sum_{k=0}^{n} \frac{u_{k+1}-u_{k}}{\Delta t}-h_{k} \frac{w_{k+1}-w_{k}}{\Delta t}\right\|^{2}\right] \\
\leqslant & 2\left\|u_{0}\right\|^{2}+2 T \sum_{k=0}^{n} \Delta t E\left[\left\|\frac{u_{k+1}-u_{k}}{\Delta t}-h_{k} \frac{w_{k+1}-w_{k}}{\Delta t}\right\|^{2}\right] \\
\leqslant & C,
\end{aligned}
$$

which proves that $\left\|\tilde{u}^{\Delta t}-\tilde{B}^{\Delta t}\right\|_{L^{\infty}\left(0, T ; L^{2}(\Omega \times D)\right)} \leq C$. Let us now show that $\left\|\nabla\left(\tilde{u}^{\Delta t}-\tilde{B}^{\Delta t}\right)\right\|_{L^{2}(\Omega \times Q)}$ is bounded independently of $\Delta t$. Using (2.5), it remains to prove that $\nabla \tilde{B}^{\Delta t}$ is bounded in $L^{2}(\Omega \times Q)$. Due to Lemma 2.3 
and the fact that $E\left[\left(w_{j+1}-w_{j}\right)^{2}\right]=\Delta t$ for any $j \in\{0, \ldots, N-1\}$, one has

$$
\begin{aligned}
\left\|\nabla \tilde{B}^{\Delta t}\right\|_{L^{2}(\Omega \times Q)}^{2} & \leqslant \Delta t \sum_{k=0}^{N} E\left\|\nabla B_{k}\right\|_{L^{2}(D)}^{2} \\
& =\Delta t \sum_{k=0}^{N} E\left[\int_{D}\left(\sum_{j=0}^{k}\left(w_{j+1}-w_{j}\right) \nabla h_{j}\right)^{2} d x\right] \\
& =\Delta t \sum_{k=0}^{N} \sum_{j=0}^{k} \int_{D} E\left[\left(\left(w_{j+1}-w_{j}\right) \nabla h_{j}\right)^{2}\right] d x \\
& =\Delta t \sum_{k=0}^{N} \sum_{j=0}^{N} \int_{D} E\left[\left(w_{j+1}-w_{j}\right)^{2}\right] E\left[\left(\nabla h_{j}\right)^{2}\right] d x \\
& =\Delta t \sum_{k=0}^{N} \Delta t E \sum_{j=0}^{k}\left\|h_{j}\right\|_{H^{1}(D)}^{2} \\
& \leqslant C .
\end{aligned}
$$

and the result holds. Finally, using the fact that $\tilde{u}^{\Delta t}-\tilde{B}^{\Delta t}$ and $\tilde{B}^{\Delta t}$ are bounded in $L^{2}(\Omega \times Q)$, one gets that $\tilde{u}^{\Delta t}$ is also bounded in $L^{2}(\Omega \times Q)$. Combining this with (2.5), one obtains the boundedness of $\tilde{u}^{\Delta t}$ in $L^{2}((0, T) \times$ $\left.\Omega, H^{1}(D)\right)$. Thanks to $(2.6)-(2.7)$, one gets the same result for $\tilde{u}^{\Delta t}(.-\Delta t)$ and $u^{\Delta t}$ which gives (2.11). Finally, (2.12) holds by noticing that $\tilde{u}^{\Delta t}(.-\Delta t)$ belongs to $\mathcal{N}_{w}^{2}\left(0, T, H^{1}(D)\right)$ as a continuous and adapted process.

\subsection{At the limit}

From the previous subsection, one gets naturally the following convergence results:

Proposition 2.9. Up to subsequences denoted in the same way, there exist $u$ belonging to $\mathcal{N}_{w}^{2}\left(0, T, H^{1}(D)\right) \cap L^{2}\left(\Omega, \mathscr{C}\left([0, T], L^{2}(D)\right)\right)$ and $\chi$ in $L^{2}(\Omega \times Q)$ such that

(i) $\quad \tilde{u}^{\Delta t}, u^{\Delta t} \rightarrow u$ in $L^{2}\left((0, T) \times \Omega, H^{1}(D)\right)$,

(ii) $\nabla \tilde{u}^{\Delta t}, \nabla u^{\Delta t} \stackrel{*}{\rightarrow} \nabla u$ in $L^{\infty}\left(0, T ; L^{2}(\Omega \times D)\right)$,

(iii) $\quad \alpha\left(\partial_{t}\left(\tilde{u}^{\Delta t}-\tilde{B}^{\Delta t}\right)\right) \rightarrow \chi$ in $L^{2}(\Omega \times Q)$,

(iv) $\quad \tilde{u}^{\Delta t}-\tilde{B}^{\Delta t} \rightarrow u-\int_{0}^{\cdot} h d w$ in $L^{2}\left(\Omega, H^{1}(Q)\right)$,

(v) $u(0,)=.u_{0}$ in $H^{1}(D)$.

Proof.

(i) Thanks to (2.6)-(2.7)-(2.11) and (2.12), there exists $u$ in $\mathcal{N}_{w}^{2}\left(0, T, H^{1}(D)\right)$ such that, up to subsequences denoted in the same way, we have

$$
\tilde{u}^{\Delta t}, u^{\Delta t}, \tilde{u}^{\Delta t}(.-\Delta t) \rightarrow u \text { in } L^{2}\left((0, T) \times \Omega, H^{1}(D)\right) .
$$

Since $\tilde{u}^{\Delta t}(.-\Delta t)$ belongs to the Hilbert space $\mathcal{N}_{w}^{2}\left(0, T, H^{1}(D)\right)$ endowed with the norm of $L^{2}\left((0, T) \times \Omega, H^{1}(D)\right)$, one gets that $u$ is also in $\mathcal{N}_{w}^{2}\left(0, T, H^{1}(D)\right)$. 
(ii) Using (2.5)-(2.6), one gets directly that up to subsequences denoted in the same way,

$$
\nabla \tilde{u}^{\Delta t}, \nabla u^{\Delta t} \stackrel{*}{\rightarrow} \nabla u \text { in } L^{\infty}\left(0, T ; L^{2}(\Omega \times D)\right) .
$$

( iii) Due to the Lipschitz property of $\alpha$ and (2.8), the sequence $\alpha\left(\partial_{t}\left(\tilde{u}^{\Delta t}-\right.\right.$ $\left.\left.\tilde{B}^{\Delta t}\right)\right)$ is bounded in $L^{2}(\Omega \times Q)$ and there exists $\chi$ in the same space such that, up to a subsequence

$$
\alpha\left(\partial_{t}\left(\tilde{u}^{\Delta t}-\tilde{B}^{\Delta t}\right)\right) \rightarrow \chi \text { in } L^{2}(\Omega \times Q) .
$$

(iv) Thanks to (2.8)-(2.9)-(2.10), there exists $\zeta$ in $L^{\infty}\left(0, T ; L^{2}(\Omega \times D)\right)$ and $L^{2}\left(\Omega, H^{1}(Q)\right)$ such that, up to a subsequence,

$\tilde{u}^{\Delta t}-\tilde{B}^{\Delta t} \rightarrow \zeta$ in $L^{2}\left(\Omega, H^{1}(Q)\right)$ and $\tilde{u}^{\Delta t}-\tilde{B}^{\Delta t} \stackrel{*}{\rightarrow} \zeta$ in $L^{\infty}\left(0, T ; L^{2}(\Omega \times D)\right)$.

Using $(i)$ and Proposition 2.5, one gets by uniqueness of the limit that $\zeta=$ $u-\int_{0}^{\cdot} h d w$.

(v) Since $L^{2}\left(\Omega, H^{1}(Q)\right)$ is continuously embedded in $L^{2}\left(\Omega, \mathscr{C}\left([0, T], L^{2}(D)\right)\right)$, one gets that $u-\int_{0} h d w$ belongs to $L^{2}\left(\Omega, \mathscr{C}\left([0, T], L^{2}(D)\right)\right)$. Moreover, as the Itô integral of an $\mathcal{N}_{w}^{2}\left(0, T, L^{2}(D)\right)$ process is a continuous square integrable $L^{2}(D)$-valued martingale (see [11]), $\int_{0} h d w$ is in $L^{2}\left(\Omega, \mathscr{C}\left([0, T], L^{2}(D)\right)\right)$. Thus $u$ belongs to $L^{2}\left(\Omega, \mathscr{C}\left([0, T], L^{2}(D)\right)\right)$ and finally $u$ is an element of $\mathscr{C}\left([0, T], L^{2}(\Omega \times D)\right)$. Particularly, we have

$$
u_{0}=\tilde{u}^{\Delta t}(0)-\tilde{B}^{\Delta t}(0) \rightarrow\left(u-\int_{0}^{\cdot} h d w\right)(0) \text { in } L^{2}(D)
$$

and so $u(0,)=.u_{0} \in H^{1}(D)$.

Using these convergence results, passing to the limit in (2.4) with respect to $\Delta t$ is now possible but the remaining difficulty is in identifying the weak limit $\chi$ in $L^{2}(\Omega \times Q)$ of $\alpha\left(\partial_{t}\left(\tilde{u}^{\Delta t}-\tilde{B}^{\Delta t}\right)\right)$. To do so, we suppose in a first step (only for technical reason) that $h$ belongs to $\mathcal{N}_{w}^{2}\left(0, T, H^{2}(D)\right)$. In a second step, we will see how to get back a solution with the announced hypothesis $h$ in $\mathcal{N}_{w}^{2}\left(0, T, H^{1}(D)\right)$.

We consider our discrete variational problem (2.1) for any $n$ in $\{0, \ldots, N-1\}$ with the test function $\frac{U_{n+1}-U_{n}}{\Delta t}$ where $U_{n+1}=u_{n+1}-\sum_{k=0}^{n}\left(w_{k+1}-w_{k}\right) h_{k}$. 
One gets $P$-a.s in $\Omega$ :

$$
\begin{aligned}
& \int_{D}\left(\frac{U_{n+1}-U_{n}}{\Delta t}\right)^{2} d x+\int_{D} \alpha\left(\frac{U_{n+1}-U_{n}}{\Delta t}\right) \frac{U_{n+1}-U_{n}}{\Delta t} d x \\
& +\int_{D} \nabla U_{n+1} \cdot \nabla\left(\frac{U_{n+1}-U_{n}}{\Delta t}\right) d x \\
= & \sum_{k=0}^{n}\left(w_{k+1}-w_{k}\right) \int_{D} \Delta h_{k} \frac{U_{n+1}-U_{n}}{\Delta t} d x+\int_{D} g_{n} \frac{U_{n+1}-U_{n}}{\Delta t} d x,
\end{aligned}
$$

and then

$$
\begin{aligned}
& \Delta t \int_{D}\left(\frac{U_{n+1}-U_{n}}{\Delta t}\right)^{2} d x+\Delta t \int_{D} \alpha\left(\frac{U_{n+1}-U_{n}}{\Delta t}\right) \frac{U_{n+1}-U_{n}}{\Delta t} d x \\
& +\frac{1}{2}\left\|\nabla U_{n+1}\right\|^{2}-\frac{1}{2}\left\|\nabla U_{n}\right\|^{2} \\
\leq & \Delta t \int_{D} \Delta B_{n+1} \frac{U_{n+1}-U_{n}}{\Delta t} d x+\Delta t \int_{D} g_{n} \frac{U_{n+1}-U_{n}}{\Delta t} d x .
\end{aligned}
$$

Now, by adding from $n=0$ to $N-1$, we obtain

$$
\begin{aligned}
& \quad \Delta t \sum_{n=0}^{N-1} \int_{D}\left(\frac{U_{n+1}-U_{n}}{\Delta t}\right)^{2} d x+\Delta t \sum_{n=0}^{N-1} \int_{D} \alpha\left(\frac{U_{n+1}-U_{n}}{\Delta t}\right) \frac{U_{n+1}-U_{n}}{\Delta t} d x \\
& +\frac{1}{2} \sum_{n=0}^{N-1}\left(\left\|\nabla U_{n+1}\right\|^{2}-\left\|\nabla U_{n}\right\|^{2}\right) \\
& \leq \Delta t \sum_{n=0}^{N-1} \int_{D} \Delta B_{n+1} \frac{U_{n+1}-U_{n}}{\Delta t} d x+\Delta t \sum_{n=0}^{N-1} \int_{D} g_{n} \frac{U_{n+1}-U_{n}}{\Delta t} d x,
\end{aligned}
$$

and this gives

$$
\begin{aligned}
& \int_{Q}\left|\partial_{t}\left(\tilde{u}^{\Delta t}-\tilde{B}^{\Delta t}\right)\right|^{2} d t d x+\int_{Q} \alpha\left(\partial_{t}\left(\tilde{u}^{\Delta t}-\tilde{B}^{\Delta t}\right)\right) \partial_{t}\left(\tilde{u}^{\Delta t}-\tilde{B}^{\Delta t}\right) d t d x \\
& +\frac{1}{2}\left(\left\|\nabla U_{N}\right\|^{2}-\left\|\nabla U_{0}\right\|^{2}\right) \\
\leq & \int_{Q} \Delta B^{\Delta t} \partial_{t}\left(\tilde{u}^{\Delta t}-\tilde{B}^{\Delta t}\right) d t d x+\int_{Q} g_{\Delta t} \partial_{t}\left(\tilde{u}^{\Delta t}-\tilde{B}^{\Delta t}\right) d t d x .
\end{aligned}
$$

Noticing that $\nabla \tilde{U}^{\Delta t}(T)=\nabla U_{N}$, we finally get after taking the expectation

$$
\begin{aligned}
& E\left[\int_{Q}\left|\partial_{t}\left(\tilde{u}^{\Delta t}-\tilde{B}^{\Delta t}\right)\right|^{2} d t d x\right]+\frac{1}{2} E\left[\|\left.\nabla \tilde{U}^{\Delta t}(T)\right|^{2}\right]-\frac{1}{2} E\left[\left\|\nabla U_{0}\right\|^{2}\right] \\
& +E\left[\int_{Q} \alpha\left(\partial_{t}\left(\tilde{u}^{\Delta t}-\tilde{B}^{\Delta t}\right)\right) \partial_{t}\left(\tilde{u}^{\Delta t}-\tilde{B}^{\Delta t}\right) d t d x\right] \\
\leq & E\left[\int_{Q} \Delta B^{\Delta t} \partial_{t}\left(\tilde{u}^{\Delta t}-\tilde{B}^{\Delta t}\right) d t d x\right]+E\left[\int_{Q} g_{\Delta t} \partial_{t}\left(\tilde{u}^{\Delta t}-\tilde{B}^{\Delta t}\right) d t d x\right] .
\end{aligned}
$$


Proposition 2.10. Up to a subsequence

$$
\alpha\left(\partial_{t}\left(\tilde{u}^{\Delta t}-\tilde{B}^{\Delta t}\right)\right) \rightarrow \alpha\left(\partial_{t}\left(u-\int_{0} h d w\right)\right) \text { in } L^{2}(\Omega \times Q) .
$$

Proof. Passing to the superior limit in (2.14), we have using Proposition 2.9

$$
\begin{aligned}
& \lim \inf _{\Delta t \rightarrow 0}\left\|\partial_{t}\left(\tilde{u}^{\Delta t}-\tilde{B}^{\Delta t}\right)\right\|_{L^{2}(\Omega \times Q)}^{2}+\frac{1}{2} \lim _{\Delta t \rightarrow 0} \inf _{\Delta} E\left[\left\|\nabla \tilde{U}^{\Delta t}(T)\right\|^{2}\right] \\
& +\lim \sup _{\Delta t \rightarrow 0} E\left[\int_{Q} \alpha\left(\partial_{t}\left(\tilde{u}^{\Delta t}-\tilde{B}^{\Delta t}\right)\right) \partial_{t}\left(\tilde{u}^{\Delta t}-\tilde{B}^{\Delta t}\right) d t d x\right]-\frac{1}{2} E\left[\left\|\nabla u_{0}\right\|^{2}\right] \\
& \leqslant E\left[\int_{Q} \int_{0}^{t} \Delta h d w \partial_{t}\left(u-\int_{0}^{t} h d w\right) d t d x\right]+E\left[\int_{Q} g \partial_{t}\left(u-\int_{0}^{t} h d w\right) d t d x\right] .
\end{aligned}
$$

Indeed, due to Remark $2.6, B^{\Delta t}$ converges strongly in $L^{2}\left((0, T) \times \Omega, H^{2}(D)\right)$ to $\int_{0}^{\cdot} h d w$ and so, by continuity of the Laplace operator, $\Delta B^{\Delta t}$ converges strongly in $L^{2}(\Omega \times Q)$ to $\Delta \int_{0} h d w$. Moreover, following [16] (Lemma 2.4.1 p.35), $\Delta \int_{0} h d w=\int_{0} \Delta h d w$.

Now, using the following embedding ([15] Lemme 8.1 p.297)

$L^{\infty}\left(0, T ; L^{2}\left(\Omega, H^{1}(D)\right) \cap \mathscr{C}\left([0, T], L^{2}\left(\Omega, L^{2}(D)\right)\right) \subset \mathscr{C}_{w}\left([0, T], L^{2}\left(\Omega, H^{1}(D)\right)\right)^{\dagger}\right.$

one gets that for all time $t$ in $[0, T], \tilde{U}^{\Delta t}(t)$ belongs to $L^{2}\left(\Omega, H^{1}(D)\right)$ and $\tilde{U}^{\Delta t}(t) \rightarrow U(t)$ in $L^{2}\left(\Omega, H^{1}(D)\right)$. Then using the lower semi-continuity of the $L^{2}\left(\Omega, H^{1}(D)\right)$-norm

$$
\lim \inf _{\Delta t \rightarrow 0} E\left[\left\|\nabla \tilde{U}^{\Delta t}(T)\right\|^{2}\right] \geqslant E\left[\|\nabla U(T)\|^{2}\right] .
$$

Finally

$$
\begin{aligned}
& \left\|\partial_{t}\left(u-\int_{0} h d w\right)\right\|_{L^{2}(\Omega \times Q)}^{2}+\frac{1}{2} E\left[\|\nabla U(T)\|^{2}\right]-\frac{1}{2} E\left[\left\|\nabla u_{0}\right\|^{2}\right] \\
& +\lim \sup _{\Delta t \rightarrow 0} E\left[\int_{Q} \alpha\left(\partial_{t}\left(\tilde{u}^{\Delta t}-\tilde{B}^{\Delta t}\right)\right) \partial_{t}\left(\tilde{u}^{\Delta t}-\tilde{B}^{\Delta t}\right) d t d x\right] \\
& \leqslant E\left[\int_{Q} \int_{0}^{t} \Delta h d w \partial_{t}\left(u-\int_{0}^{t} h d w\right) d t d x\right]+E\left[\int_{Q} g \partial_{t}\left(u-\int_{0}^{t} h d w\right) d t d x\right] .
\end{aligned}
$$

Noticing that $P$-almost surely in $\Omega, U=u-\int_{0} h d w$ satisfies the heat equation

$$
\left\{\begin{aligned}
\partial_{t} U-\Delta U & =\bar{g} \\
U(0, .) & =u_{0}
\end{aligned}\right.
$$

$\dagger_{\mathscr{C}_{w}}\left([0, T], L^{2}\left(\Omega, H^{1}(D)\right)\right)$ denotes the set of functions defined on $[0, T]$ with values in $L^{2}\left(\Omega, H^{1}(D)\right)$ which are weakly continuous. 
where $\bar{g}=g-\chi+\int_{0}^{t} \Delta h d w$, one has the following energy equality (see [10] Theorem X.11 p.220), for any $t \in[0, T]$ by denoting $Q_{t}=(0, t) \times D$ :

$$
\begin{aligned}
& \int_{Q_{t}}\left|\partial_{t} U\right|^{2} d s d x+\int_{Q_{t}} \chi \partial_{t} U d s d x+\frac{1}{2}\|\nabla U(t)\|^{2} \\
= & \int_{Q_{t}} \int_{0}^{s} \Delta h d w \partial_{t} U d s d x+\int_{Q_{t}} g \partial_{t} U d s d x+\frac{1}{2}\left\|\nabla u_{0}\right\|^{2} .
\end{aligned}
$$

Then by taking the expectation :

$$
\begin{aligned}
& E\left[\int_{Q_{t}}\left|\partial_{t} U\right|^{2} d s d x\right]+E\left[\int_{Q_{t}} \chi \partial_{t} U d s d x\right]+\frac{1}{2} E\left[\|\nabla U(t)\|^{2}\right] \\
= & E\left[\int_{Q_{t}} \int_{0}^{s} \Delta h d w \partial_{t} U d s d x\right]+E\left[\int_{Q_{t}} g \partial_{t} U d s d x\right]+\frac{1}{2} E\left[\left\|\nabla u_{0}\right\|^{2}\right] .
\end{aligned}
$$

In this way, by injecting (2.16) in (2.15) we finally have

$$
\begin{aligned}
& \lim _{\Delta t \rightarrow 0} \sup _{\Delta t \rightarrow} E\left[\int_{Q} \alpha\left(\partial_{t}\left(\tilde{u}^{\Delta t}-\tilde{B}^{\Delta t}\right)\right) \partial_{t}\left(\tilde{u}^{\Delta t}-\tilde{B}^{\Delta t}\right) d t d x\right] \\
\leq & E\left[\int_{Q} \chi \partial_{t}\left(u-\int_{0} h d w\right) d t d x\right] .
\end{aligned}
$$

As $\alpha: \mathbb{R} \rightarrow \mathbb{R}$ is a Lipschitz-continuous nondecreasing function, the operator $A_{\alpha}: v \in L^{2}(\Omega \times Q) \mapsto \alpha(v) \in L^{2}(\Omega \times Q)$ is maximal monotone and one gets $\chi=\alpha\left(\partial_{t}\left(u-\int_{0}^{t} h d w\right)\right)($ see LiONS [15] p.172).

Proposition 2.11. The application $t \in[0, T] \mapsto E\left[\|\nabla u(t)\|^{2}\right] \in \mathbb{R}$ is continuous.

Proof. Using (2.16) and Lebesgue's theorem, one gets the continuity of

$$
t \in[0, T] \mapsto E\left[\|\nabla U(t)\|^{2}\right] \in \mathbb{R} .
$$

Moreover, since $\int_{0} h d w$ belongs to $\mathscr{C}\left([0, T], L^{2}\left(\Omega, H^{1}(D)\right)\right)$, thus

$$
t \in[0, T] \mapsto E\left[\left\|\int_{0}^{t} \nabla h(s) d w(s)\right\|^{2}\right] \in \mathbb{R}
$$

is continuous and one gets the announced result.

Remark 2.12. Note that $u$ belongs to $\mathscr{C}\left([0, T], L^{2}\left(\Omega, H^{1}(D)\right)\right)$. Indeed, due to $(2.16)$, Lebesgue's theorem and the fact that $U=u-\int_{0} h d w$ is an element of $\mathscr{C}\left([0, T], L^{2}\left(\Omega, L^{2}(D)\right)\right)$, one gets the continuity of the application

$$
t \in[0, T] \mapsto E\left[\|U(t)\|_{H^{1}(D)}^{2}\right] \in \mathbb{R}
$$

Combining this with the fact that $U$ also belongs to $\mathscr{C}_{w}\left([0, T], L^{2}\left(\Omega, H^{1}(D)\right)\right)$, one concludes that $U$ is in $\mathscr{C}\left([0, T], L^{2}\left(\Omega, H^{1}(D)\right)\right)$ and due to the regularity of Itô integral, it is the same for $u$. 


\subsection{Proof of Theorem 1.4}

With the study done in the previous section, we are able to show the result of existence and uniqueness of a solution for Problem (1.1) stated in Theorem 1.4. Let us begin with the uniqueness result. We consider $h$ in $\mathcal{N}_{w}^{2}\left(0, T, H^{1}(D)\right), u, \hat{u}$ two solutions in the sense of Definition 1.1 of our stochastic problem

$$
\left\{\begin{aligned}
\partial_{t}\left(u-\int_{0} h d w\right)-\Delta u+\alpha\left(\partial_{t}\left(u-\int_{0} h d w\right)\right) & =g \text { in }(0, T) \times D \times \Omega, \\
\nabla u . \mathbf{n} & =0 \text { on }(0, T) \times \partial D \times \Omega, \\
u(0, .) & =u_{0} .
\end{aligned}\right.
$$

Using the notations $U=u-\int_{0} h d w, \hat{U}=\hat{u}-\int_{0} h d w$, one has

$$
\left\{\begin{aligned}
\partial_{t}(U-\hat{U})-\Delta(U-\hat{U})+\alpha\left(\partial_{t} U\right)-\alpha\left(\partial_{t} \hat{U}\right) & =0 \text { in }(0, T) \times D \times \Omega, \\
\nabla(U-\hat{U}) \cdot \mathbf{n} & =0 \text { on }(0, T) \times \partial D \times \Omega, \\
(U-\hat{U})(0, .) & =0 .
\end{aligned}\right.
$$

This means that $U-\hat{U}$ is the solution of the heat equation

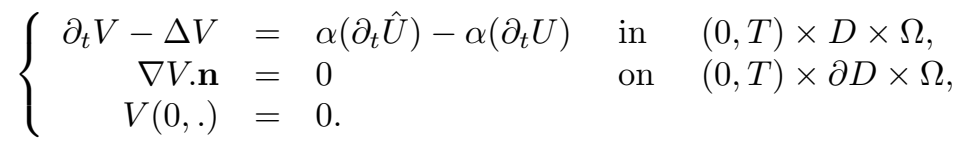

As previously one has the following energy equality for any $t$ in $[0, T]$ by denoting $Q_{t}=(0, t) \times D$ :

$$
\begin{aligned}
& E\left[\int_{Q_{t}}\left|\partial_{t}(U-\hat{U})\right|^{2} d x d t\right]+\frac{1}{2} E\left[\|\nabla(U-\hat{U})(t)\|_{L^{2}(D)}^{2}\right] \\
= & \frac{1}{2}\|\nabla(U-\hat{U})(0)\|_{L^{2}(D)}^{2}-E\left[\int_{Q_{t}}\left(\alpha\left(\partial_{t} U\right)-\alpha\left(\partial_{t} \hat{U}\right)\right) \partial_{t}(U-\hat{U}) d x d t\right],
\end{aligned}
$$

with $t \in[0, T] \mapsto E\left[\|\nabla(U-\hat{U})(t)\|^{2}\right]$ continuous.

Using the coercivity property of $\alpha$ one gets for any $t$ in $[0, T]$

$$
\left(\tilde{C}_{\alpha}+1\right)\left\|\partial_{t}(U-\hat{U})\right\|_{L^{2}\left(\Omega \times Q_{t}\right)}^{2}+\frac{1}{2} E\left[\|\nabla(U-\hat{U})(t)\|_{L^{2}(D)}^{2}\right] \leqslant 0 .
$$

Moreover, the study of the heat equation also provides the following estimate for any $t$ in $[0, T]$

$$
\begin{aligned}
& \frac{1}{2} E\left[\|(U-\hat{U})(t)\|^{2}\right]-\frac{1}{2}\|(U-\hat{U})(0)\|^{2}+\|\nabla(U-\hat{U})\|_{L^{2}\left(\Omega \times Q_{t}\right)}^{2} \\
\leqslant & \frac{1}{2}\|U-\hat{U}\|_{L^{2}\left(\Omega \times Q_{t}\right)}+\frac{C_{\alpha}^{2}}{2}\left\|\partial_{t}(U-\hat{U})\right\|_{L^{2}\left(\Omega \times Q_{t}\right)}^{2},
\end{aligned}
$$

which gives using (2.17)

$$
\frac{1}{2} E\left[\|(U-\hat{U})(t)\|^{2}\right] \leqslant \frac{1}{2}\|U-\hat{U}\|_{L^{2}\left(\Omega \times Q_{t}\right)},
$$

finally $u=\hat{u}$ and we have uniqueness of the solution. 
Now let us prove the existence result. Set $h$ in $\mathcal{N}_{w}^{2}\left(0, T, H^{2}(D)\right)$. Thanks to the previous section, for all $v$ in $H^{1}(D), \varphi$ in $L^{2}(0, T)$ and $\psi$ in $L^{\infty}(\Omega)$, the following variational equation holds

$$
\begin{aligned}
& E\left[\int_{Q} \partial_{t}\left(u-\int_{0}^{\cdot} h d w\right) v \varphi \psi d x d t\right]+E\left[\int_{Q} \nabla u \cdot \nabla v \varphi \psi d x d t\right] \\
& +E\left[\int_{Q} \alpha\left(\partial_{t}\left(u-\int_{0} h d w\right)\right) v \varphi \psi d x d t\right]=E\left[\int_{Q} g v \varphi \psi d x d t\right] .
\end{aligned}
$$

Using the separability of $H^{1}(D)$, one gets that a.e in $(0, T), P$-a.s in $\Omega$ and for any $v$ in $H^{1}(D)$

$$
\int_{D} \tilde{\alpha}\left(\partial_{t}\left(u-\int_{0}^{\cdot} h d w\right)\right) v d x+\int_{D} \nabla u \cdot \nabla v d x=\int_{Q} g v d x
$$

Using Proposition 2.9 and Remark 2.12, $u$ has the regularities required by Definition 1.1 and satisfies the initial condition $u(0,)=.u_{0} \in H^{1}(D)$. Thus we have existence of a solution $u$ in the sense of Definition 1.1.

Let us now treat the case $h \in \mathcal{N}_{w}^{2}\left(0, T, H^{1}(D)\right)$ stated by Theorem 1.4. We decide to approach $h$ by a sequence $\left(h_{n}\right)_{n} \subset \mathcal{N}_{w}^{2}\left(0, T, \mathscr{C}_{c}^{\infty}(\bar{D})\right)$. Set $n, m \in \mathbb{N}$ and consider $g_{n}, g_{m}$ in $\mathcal{N}_{w}^{2}\left(0, T, L^{2}(D)\right)$ and $u_{n, 0}, u_{m, 0}$ in $H^{1}(D)$. Then, from the previous proof, there exist two predictable processes in $\mathcal{N}_{w}^{2}\left(0, T, H^{1}(D)\right)$ denoted $u_{n}$ and $u_{m}$ satisfying the variational formulation (1.4) where the data triplet $\left(g, h, u_{0}\right)$ is replaced respectively by $\left(g_{n}, h_{n}, u_{n, 0}\right)$ and $\left(g_{m}, h_{m}, u_{m, 0}\right)$. Moreover, $u_{n}$ and $u_{m}$ possess the following regularities

$$
\begin{aligned}
& u_{n}, u_{m} \in L^{2}\left(\Omega, \mathscr{C}\left([0, T], L^{2}(D)\right)\right) \cap \mathscr{C}\left([0, T], L^{2}\left(\Omega, H^{1}(D)\right)\right), \\
& u_{n}-\int_{0} h_{n} d w, u_{m}-\int_{0} h_{m} d w \in L^{2}\left(\Omega, H^{1}(Q)\right) \cap L^{\infty}\left(0, T ; L^{2}\left(\Omega, H^{1}(D)\right)\right) .
\end{aligned}
$$

Using the notations $U_{n}=u_{n}-\int_{0}^{\cdot} h_{n} d w$ and $U_{m}=u_{m}-\int_{0}^{\cdot} h_{m} d w$ we have $t$-almost everywhere in $(0, T), P$-almost surely in $\Omega$ and for any $v$ in $H^{1}(D)$

$$
\begin{aligned}
& \int_{D} \partial_{t}\left(U_{n}-U_{m}\right) v d x+\int_{D}\left(\alpha\left(\partial_{t}\left(U_{n}\right)\right)-\alpha\left(\partial_{t}\left(U_{m}\right)\right)\right) v d x \\
& +\int_{D} \nabla\left(u_{n}-u_{m}\right) \cdot \nabla v d x=\int_{D}\left(g_{n}-g_{m}\right) v d x .
\end{aligned}
$$

For a fixed $t$ in $[0, T]$, by taking the test function

$$
v=\frac{\left(U_{n}-U_{m}\right)(t)-\left(U_{n}-U_{m}\right)(t-\Delta t)}{\Delta t}
$$


in (2.18), we get

$$
\begin{aligned}
& \int_{D} \partial_{t}\left(U_{n}-U_{m}\right) \frac{\left(U_{n}-U_{m}\right)(t)-\left(U_{n}-U_{m}\right)(t-\Delta t)}{\Delta t} d x \\
& +\frac{1}{\Delta t} \int_{D} \nabla\left(u_{n}(t)-u_{m}(t)\right) \cdot \nabla\left(u_{n}(t)-u_{m}(t)-u_{n}(t-\Delta t)-u_{m}(t-\Delta t)\right) d x \\
& -\frac{1}{\Delta t} \int_{D} \nabla\left(u_{n}(t)-u_{m}(t)\right) \cdot \nabla\left(\int_{t-\Delta t}^{t}\left(h_{n}-h_{m}\right) d w\right) d x \\
& +\int_{D}\left(\alpha\left(\partial_{t} U_{n}\right)-\alpha\left(\partial_{t} U_{m}\right)\right) \frac{\left(U_{n}-U_{m}\right)(t)-\left(U_{n}-U_{m}\right)(t-\Delta t)}{\Delta t} d x \\
& =\int_{D}\left(g_{n}-g_{m}\right) \frac{\left(U_{n}-U_{m}\right)(t)-\left(U_{n}-U_{m}\right)(t-\Delta t)}{\Delta t} d x
\end{aligned}
$$

Moreover, by noticing that

$$
\begin{aligned}
& \int_{D} \nabla\left(u_{n}(t)-u_{m}(t)\right) \cdot \nabla\left(u_{n}(t)-u_{m}(t)-u_{n}(t-\Delta t)-u_{m}(t-\Delta t)\right) d x \\
= & \frac{1}{2}\left[\left\|\nabla\left(u_{n}-u_{m}\right)(t)\right\|^{2}-\left\|\nabla\left(u_{n}-u_{m}\right)(t-\Delta t)\right\|^{2}\right. \\
& \left.+\left\|\nabla\left(u_{n}-u_{m}\right)-\nabla\left(u_{n}-u_{m}\right)(t-\Delta t)\right\|^{2}\right],
\end{aligned}
$$

we obtain

$$
\begin{aligned}
& \int_{D} \partial_{t}\left(U_{n}-U_{m}\right) \frac{\left(U_{n}-U_{m}\right)(t)-\left(U_{n}-U_{m}\right)(t-\Delta t)}{\Delta t} d x \\
& \frac{1}{2 \Delta t}\left[\left\|\nabla\left(u_{n}-u_{m}\right)(t)\right\|^{2}-\left\|\nabla\left(u_{n}-u_{m}\right)(t-\Delta t)\right\|^{2}\right. \\
+ & \left.\left\|\nabla\left(u_{n}-u_{m}\right)(t)-\nabla\left(u_{n}-u_{m}\right)(t-\Delta t)\right\|^{2}\right] \\
- & \frac{1}{\Delta t} \int_{D} \nabla\left(\left(u_{n}-u_{m}\right)(t)-\left(u_{n}-u_{m}\right)(t-\Delta t)\right) \cdot \nabla\left(\int_{t-\Delta t}^{t}\left(h_{n}-h_{m}\right) d w\right) d x \\
- & \frac{1}{\Delta t} \int_{D} \nabla\left(\left(u_{n}-u_{m}\right)(t-\Delta t)\right) \cdot \nabla\left(\int_{t-\Delta t}^{t}\left(h_{n}-h_{m}\right) d w\right) d x \\
+ & \int_{D}\left(\alpha\left(\partial_{t} U_{n}\right)-\alpha\left(\partial_{t} U_{m}\right)\right) \frac{\left(U_{n}-U_{m}\right)(t)-\left(U_{n}-U_{m}\right)(t-\Delta t)}{\Delta t} d x \\
= & \int_{D}\left(g_{n}-g_{m}\right) \frac{\left(U_{n}-U_{m}\right)(t)-\left(U_{n}-U_{m}\right)(t-\Delta t)}{\Delta t} d x .
\end{aligned}
$$


Thus

$$
\begin{aligned}
& \int_{D} \partial_{t}\left(U_{n}-U_{m}\right) \frac{\left(U_{n}-U_{m}\right)(t)-\left(U_{n}-U_{m}\right)(t-\Delta t)}{\Delta t} d x \\
& +\frac{1}{2 \Delta t}\left[\left\|\nabla\left(u_{n}-u_{m}\right)(t)\right\|^{2}-\left\|\nabla\left(u_{n}-u_{m}\right)(t-\Delta t)\right\|^{2}\right] \\
& +\frac{1}{4 \Delta t}\left[\left\|\nabla\left(u_{n}-u_{m}\right)(t)-\nabla\left(u_{n}-u_{m}\right)(t-\Delta t)\right\|^{2}\right. \\
& -4\left\|\nabla \int_{t-\Delta t}^{t}\left(h_{n}-h_{m}\right) d w\right\|^{2} \\
& \left.+\left\|\nabla\left(\left(u_{n}-u_{m}\right)(t)-\left(u_{n}-u_{m}\right)(t-\Delta t)-2 \int_{t-\Delta t}^{t}\left(h_{n}-h_{m}\right) d w\right)\right\|^{2}\right] \\
& -\frac{1}{\Delta t} \int_{D} \nabla\left(u_{n}-u_{m}\right)(t-\Delta t) \cdot \nabla\left(\int_{t-\Delta t}^{t}\left(h_{n}-h_{m}\right) d w\right) d x \\
& +\int_{D}\left(\alpha\left(\partial_{t} U_{n}\right)-\alpha\left(\partial_{t} U_{m}\right)\right) \frac{\left(U_{n}-U_{m}\right)(t)-\left(U_{n}-U_{m}\right)(t-\Delta t)}{\Delta t} d x \\
& =\int_{D}\left(g_{n}-g_{m}\right) \frac{\left(U_{n}-U_{m}\right)(t)-\left(U_{n}-U_{m}\right)(t-\Delta t)}{\Delta t} d x .
\end{aligned}
$$

By taking the expectation, the integral from $\Delta t$ to $T$ and by using the fact that

$$
E\left[\int_{D} \nabla\left(u_{n}-u_{m}\right)(t-\Delta t) \cdot \nabla\left(\int_{t-\Delta t}^{t}\left(h_{n}-h_{m}\right) d w\right) d x\right]=0
$$

one gets

$$
\begin{aligned}
& \int_{\Delta t}^{T} E\left[\int_{D} \partial_{t}\left(U_{n}-U_{m}\right) \frac{\left(U_{n}-U_{m}\right)(t)-\left(U_{n}-U_{m}\right)(t-\Delta t)}{\Delta t} d x\right] d t \\
+ & \frac{1}{2 \Delta t} \int_{\Delta t}^{T} E\left[\left\|\nabla\left(u_{n}-u_{m}\right)(t)\right\|^{2}-\left\|\nabla\left(u_{n}-u_{m}\right)(t-\Delta t)\right\|^{2}\right] d t \\
+ & \int_{\Delta t}^{T} E\left[\int_{D}\left(\alpha\left(\partial_{t} U_{n}\right)-\alpha\left(\partial_{t} U_{m}\right)\right) \frac{\left(U_{n}-U_{m}\right)(t)-\left(U_{n}-U_{m}\right)(t-\Delta t)}{\Delta t} d x\right] d t \\
\leq & \int_{\Delta t}^{T} \frac{1}{\Delta t} E\left[\left\|\nabla \int_{t-\Delta t}^{t}\left(h_{n}-h_{m}\right) d w\right\|^{2}\right] d t \\
& +\int_{D}\left(g_{n}-g_{m}\right) \frac{\left(U_{n}-U_{m}\right)(t)-\left(U_{n}-U_{m}\right)(t-\Delta t)}{\Delta t} d x \\
= & \int_{\Delta t}^{T} \frac{1}{\Delta t} \int_{t-\Delta t}^{t} E\left[\left\|\nabla\left(h_{n}-h_{m}\right)\right\|^{2}\right] d s d t \\
& +\int_{D}\left(g_{n}-g_{m}\right) \frac{\left(U_{n}-U_{m}\right)(t)-\left(U_{n}-U_{m}\right)(t-\Delta t)}{\Delta t} d x,
\end{aligned}
$$


and by using changes of variables

$$
\begin{aligned}
& \int_{\Delta t}^{T} E\left[\int_{D} \partial_{t}\left(U_{n}-U_{m}\right) \frac{\left(U_{n}-U_{m}\right)(t)-\left(U_{n}-U_{m}\right)(t-\Delta t)}{\Delta t} d x\right] d t \\
+ & \frac{1}{2 \Delta t} \int_{\Delta t}^{T} E\left[\left\|\nabla\left(u_{n}-u_{m}\right)(t)\right\|^{2}\right] d t-\frac{1}{2 \Delta t} \int_{0}^{\Delta t} E\left[\left\|\nabla\left(u_{n}-u_{m}\right)(t)\right\|^{2}\right] d t \\
+ & \int_{\Delta t}^{T} E\left[\int_{D}\left(\alpha\left(\partial_{t} U_{n}\right)-\alpha\left(\partial_{t} U_{m}\right)\right) \frac{\left(U_{n}-U_{m}\right)(t)-\left(U_{n}-U_{m}\right)(t-\Delta t)}{\Delta t} d x\right] d t \\
\leq & \left\|\nabla\left(h_{n}-h_{m}\right)\right\|_{L^{2}(\Omega \times Q)}^{2} \\
& +\int_{D}\left(g_{n}-g_{m}\right) \frac{\left(U_{n}-U_{m}\right)(t)-\left(U_{n}-U_{m}\right)(t-\Delta t)}{\Delta t} d x .
\end{aligned}
$$

By passing to the limit on $\Delta t$ and using Proposition 2.11 we obtain:

$$
\begin{aligned}
& E\left[\int_{Q}\left|\partial_{t}\left(U_{n}-U_{m}\right)\right|^{2} d x d t\right]+\frac{1}{2} E\left[\left\|\nabla\left(u_{n}-u_{m}\right)(T)\right\|^{2}\right] \\
+ & E\left[\int_{Q}\left(\alpha\left(\partial_{t} U_{n}\right)-\alpha\left(\partial_{t} U_{m}\right)\right) \partial_{t}\left(U_{n}-U_{m}\right) d x d t\right]-\frac{1}{2} E\left[\left\|\nabla\left(u_{n}-u_{m}\right)(0)\right\|^{2}\right] \\
\leq & \left\|\nabla\left(h_{n}-h_{m}\right)\right\|_{L^{2}(\Omega \times Q)}^{2}+\int_{D}\left(g_{n}-g_{m}\right) \partial_{t}\left(U_{n}-U_{m}\right) d x
\end{aligned}
$$

Then, due to the coercivity of $\alpha$

$$
\begin{aligned}
& \left(\tilde{C}_{\alpha}+\frac{1}{2}\right)\left\|\partial_{t}\left(U_{n}-U_{m}\right)\right\|_{L^{2}(\Omega \times Q)}^{2}+\frac{1}{2} E\left[\left\|\nabla\left(u_{n}-u_{m}\right)(T)\right\|^{2}\right] \\
\leq & \frac{1}{2} E\left[\left\|\nabla\left(u_{n}-u_{m}\right)(0)\right\|^{2}\right]+\left\|\nabla\left(h_{n}-h_{m}\right)\right\|_{L^{2}(\Omega \times Q)}^{2}+\frac{1}{2}\left\|g_{n}-g_{m}\right\|_{L^{2}(\Omega \times Q)}^{2} .
\end{aligned}
$$

Moreover, by denoting $Q_{t}=(0, t) \times D$, one also has for any $t \in[0, T]$

$$
\begin{aligned}
& \left(\tilde{C}_{\alpha}+\frac{1}{2}\right)\left\|\partial_{t}\left(U_{n}-U_{m}\right)\right\|_{L^{2}\left(\Omega \times Q_{t}\right)}^{2}+\frac{1}{2} E\left[\left\|\nabla\left(u_{n}-u_{m}\right)(t)\right\|^{2}\right] \\
\leqslant & \frac{1}{2} E\left[\left\|\nabla\left(u_{n}-u_{m}\right)(0)\right\|^{2}\right]+\left\|\nabla\left(h_{n}-h_{m}\right)\right\|_{L^{2}\left(\Omega \times Q_{t}\right)}^{2}+\frac{1}{2}\left\|g_{n}-g_{m}\right\|_{L^{2}\left(\Omega \times Q_{t}\right)}^{2} .
\end{aligned}
$$

In the same manner, using the test function $U_{n}-U_{m}$ in $(2.18)$, one shows the following inequality for any $t \in[0, T]$

$$
\begin{aligned}
& \frac{1}{2} E\left[\left\|\left(U_{n}-U_{m}\right)(t)\right\|^{2}\right]-\frac{1}{2} E\left[\left\|\left(U_{n}-U_{m}\right)(0)\right\|^{2}\right]+\frac{1}{2}\left\|\nabla\left(u_{n}-u_{m}\right)\right\|_{L^{2}\left(\Omega \times Q_{t}\right)}^{2} \\
\leqslant & C_{\alpha}^{2}\left\|\partial_{t}\left(U_{n}-U_{m}\right)\right\|_{L^{2}\left(\Omega \times Q_{t}\right)}^{2}+\frac{1}{2}\left\|U_{n}-U_{m}\right\|_{L^{2}\left(\Omega \times Q_{t}\right)}^{2} \\
& +\frac{T}{2}\left\|\nabla\left(h_{n}-h_{m}\right)\right\|_{L^{2}\left(\Omega \times Q_{t}\right)}^{2}+\left\|g_{n}-g_{m}\right\|_{L^{2}\left(\Omega \times Q_{t}\right)}^{2} .
\end{aligned}
$$


Moreover, due to (2.20):

$$
\begin{aligned}
\left\|\partial_{t}\left(U_{n}-U_{m}\right)\right\|_{L^{2}\left(\Omega \times Q_{t}\right)}^{2} \leqslant & \frac{1}{\tilde{C}_{\alpha}+\frac{1}{2}}\left\{\frac{1}{2} E\left[\left\|\nabla\left(u_{n}-u_{m}\right)(0)\right\|^{2}\right]\right. \\
& \left.+\left\|\nabla\left(h_{n}-h_{m}\right)\right\|_{L^{2}\left(\Omega \times Q_{t}\right)}^{2}+\frac{1}{2}\left\|g_{n}-g_{m}\right\|_{L^{2}\left(\Omega \times Q_{t}\right)}^{2}\right\},
\end{aligned}
$$

and thus

$$
\begin{aligned}
& E\left[\left\|\left(U_{n}-U_{m}\right)(t)\right\|^{2}\right] \\
\leqslant & \left\|\left(u_{n}-u_{m}\right)(0)\right\|^{2}+T\left\|\nabla\left(h_{n}-h_{m}\right)\right\|_{L^{2}\left(\Omega \times Q_{t}\right)}^{2}+2\left\|g_{n}-g_{m}\right\|_{L^{2}\left(\Omega \times Q_{t}\right)}^{2} \\
& +\left\|U_{n}-U_{m}\right\|_{L^{2}\left(\Omega \times Q_{t}\right)}^{2}+\frac{2 C_{\alpha}^{2}}{\tilde{C}_{\alpha}+\frac{1}{2}}\left\{\frac{1}{2} E\left[\left\|\nabla\left(u_{n}-u_{m}\right)(0)\right\|^{2}\right]\right. \\
& \left.+\left\|\nabla\left(h_{n}-h_{m}\right)\right\|_{L^{2}\left(\Omega \times Q_{t}\right)}^{2}+\frac{1}{2}\left\|g_{n}-g_{m}\right\|_{L^{2}\left(\Omega \times Q_{t}\right)}^{2}\right\} \\
\leqslant & K_{\alpha}^{n, m}+\int_{0}^{t} E\left[\left\|\left(U_{n}-U_{m}\right)(s)\right\|^{2}\right] d s,
\end{aligned}
$$

where

$$
\begin{aligned}
K_{\alpha}^{n, m}= & \left\|\left(u_{n}-u_{m}\right)(0)\right\|^{2}+\left(\frac{2 C_{\alpha}^{2}}{\tilde{C}_{\alpha}+\frac{1}{2}}+T+4\right)\left\{\frac{1}{2} E\left[\left\|\nabla\left(u_{n}-u_{m}\right)(0)\right\|^{2}\right]\right. \\
& \left.+\left\|\nabla\left(h_{n}-h_{m}\right)\right\|_{L^{2}\left(\Omega \times Q_{t}\right)}^{2}+\frac{1}{2}\left\|g_{n}-g_{m}\right\|_{L^{2}\left(\Omega \times Q_{t}\right)}^{2}\right\} .
\end{aligned}
$$

Grönwall's Lemma then asserts that for any $t$ in $[0, T]$

$$
E\left[\left\|\left(U_{n}-U_{m}\right)(t)\right\|^{2}\right] \leqslant K_{\alpha}^{n, m} e^{t} .
$$

By taking $\left(u_{n, 0}, g_{n}\right)=\left(u_{m, 0}, g_{m}\right)$ in (2.20) and (2.21), one gets the estimates

$$
\begin{aligned}
\left(\tilde{C}_{\alpha}+\frac{1}{2}\right)\left\|\partial_{t}\left(U_{n}-U_{m}\right)\right\|_{L^{2}(\Omega \times Q)}^{2} & +\frac{1}{2} \sup _{t \in[0, T]} E\left[\left\|\nabla\left(u_{n}-u_{m}\right)(t)\right\|^{2}\right] \\
& \leqslant\left\|\nabla\left(h_{n}-h_{m}\right)\right\|_{L^{2}(\Omega \times Q)}^{2},
\end{aligned}
$$

and $\sup _{t \in[0, T]} E\left[\left\|\left(U_{n}-U_{m}\right)(t)\right\|^{2}\right] \leqslant\left(\frac{2 C_{\alpha}^{2}}{\tilde{C}_{\alpha}+\frac{1}{2}}+T\right)\left\|\nabla\left(h_{n}-h_{m}\right)\right\|_{L^{2}(\Omega \times Q)}^{2} e^{T}$.

Since $\left(h_{n}\right)_{n}$ is a Cauchy sequence in $\mathcal{N}_{w}^{2}\left(0, T, H^{1}(D)\right),\left(U_{n}\right)_{n}$ and $\left(u_{n}\right)_{n}$ are also Cauchy sequences respectively in $L^{2}\left(\Omega, W\left(0, T, H^{1}(D), L^{2}(D)\right)\right)^{\dagger}$ and $\mathscr{C}\left([0, T], L^{2}\left(\Omega, H^{1}(D)\right)\right) \cap \mathcal{N}_{w}^{2}\left(0, T, H^{1}(D)\right)$.

As mentioned by DA Prato-ZABCZYK $[11], \mathcal{N}_{w}^{2}\left(0, T, H^{1}(D)\right)$ is complete, and there exists $u$ in $\mathcal{N}_{w}^{2}\left(0, T, H^{1}(D)\right)$ such that

$$
\begin{aligned}
u_{n} \rightarrow u & \text { in } & \mathcal{N}_{w}^{2}\left(0, T, H^{1}(D)\right), \\
U_{n} \rightarrow u-\int_{0} h d w & \text { in } & L^{2}\left(\Omega, W\left(0, T, H^{1}(D), L^{2}(D)\right)\right) .
\end{aligned}
$$

${ }^{\dagger} W\left(0, T, H^{1}(D), L^{2}(D)\right)=\left\{v \in L^{2}\left(0, T, H^{1}(D)\right)\right.$ such that $\left.\partial_{t} v \in L^{2}\left(0, T, L^{2}(D)\right)\right\}$. 
Moreover, since $U_{n}(0,$.$) converges to u(0,$.$) in L^{2}(\Omega \times D)$, we obtain that $u_{0}=u(0,$.$) . Finally, we get that t$-almost everywhere in $(0, T), P$-almost surely in $\Omega$ and for any $v$ in $H^{1}(D)$

$\int_{D} \partial_{t}\left(u-\int_{0}^{\cdot} h d w\right) v d x+\int_{D} \nabla u . \nabla v d x+\int_{D} \alpha\left(\partial_{t}\left(u-\int_{0}^{\cdot} h d w\right)\right) v d x=\int_{D} g v d x$

and we have the existence result when $h \in \mathcal{N}_{w}^{2}\left(0, T, H^{1}(D)\right)$ as announced in Theorem 1.4.

\subsection{Proof of Proposition 1.5}

Let us show the continuous dependence of the solution with respect to the data. The idea is to use the same arguments as previously. Consider $g, \hat{g}$ in $\mathcal{N}_{w}^{2}\left(0, T, L^{2}(D)\right), h, \hat{h}$ in $\mathcal{N}_{w}^{2}\left(0, T, H^{1}(D)\right), u_{0}, \hat{u}_{0}$ in $H^{1}(D)$ and denote by $u$, $\hat{u}$ the associated solutions to Problem (1.1) in the sense of Definition 1.1 with the respective sets of data $\left(g, h, u_{0}\right)$ and $\left(\hat{g}, \hat{h}, \hat{u}_{0}\right)$. Additionally, we consider two sequences $\left(h_{n}\right)_{n},\left(\hat{h}_{n}\right)_{n}$ belonging to $\mathcal{N}_{w}^{2}\left(0, T, \mathscr{C}_{c}^{\infty}(\bar{D})\right)$ as regularizations of $h$ and $\hat{h}$. Then there exist two processes $u_{n}$ and $\hat{u}_{n}$ satisfying respectively the following problems in the sense of Definition 1.1

$$
\left\{\begin{array}{rll}
\tilde{\alpha}\left(\partial_{t}\left(u_{n}-\int_{0} h_{n} d w\right)\right)-\Delta u_{n}=g & \text { in } & (0, T) \times D \times \Omega, \\
\tilde{\alpha}\left(\partial_{t}\left(\hat{u}_{n}-\int_{0} \hat{h}_{n} d w\right)\right)-\Delta \hat{u}_{n}=\hat{g} & \text { in } \quad & (0, T) \times D \times \Omega, \\
\nabla u_{n} \cdot \mathbf{n}=\nabla \hat{u}_{n} \cdot \mathbf{n}=0 & \text { on } \quad & (0, T) \times \partial D \times \Omega, \\
u_{n}(0, .)=u_{0} & \text { and } \quad & \hat{u}_{n}(0, .)=\hat{u}_{0} .
\end{array}\right.
$$

Reasoning as for the existence proof, and by denoting $U_{n}=u_{n}-\int_{0} h_{n} d w$ and $\hat{U}_{n}=\hat{u}_{n}-\int_{0} \hat{h}_{n} d w$, we prove that $\left(u_{n}\right),\left(\hat{u}_{n}\right)$ are Cauchy sequences in $\mathcal{N}_{w}^{2}\left(0, T, H^{1}(D)\right) \cap \mathscr{C}\left([0, T], L^{2}\left(\Omega, H^{1}(D)\right)\right)$ and that $\left(U_{n}\right),\left(\hat{U}_{n}\right)$ are Cauchy sequences in $L^{2}\left(\Omega, W\left(0, T, H^{1}(D), L^{2}(D)\right)\right)$. Due to the uniqueness of the solution of (1.1), one concludes that $\left(u_{n}\right)_{n}$ and $\left(\hat{u}_{n}\right)_{n}$ converge respectively to the solutions $u$ and $\hat{u}$, both in $\mathcal{N}_{w}^{2}\left(0, T, H^{1}(D)\right)$ and $\mathscr{C}\left([0, T], L^{2}\left(\Omega, H^{1}(D)\right)\right)$. Moreover, one shows also that $\left(\partial_{t} U_{n}\right)_{n}$ and $\left(\partial_{t} \hat{U}_{n}\right)_{n}$ converge in $L^{2}(\Omega \times Q)$ respectively to $\partial_{t} U=\partial_{t}\left(u-\int_{0} h d w\right)$ and $\partial_{t} \hat{U}=\partial_{t}\left(\hat{u}-\int_{0} \hat{h} d w\right)$. As for the obtention of (2.20)-(2.21), one shows that for any $t$ in $[0, T]$,

$$
\begin{aligned}
& \left(\tilde{C}_{\alpha}+\frac{1}{2}\right)\left\|\partial_{t}\left(U_{n}-\hat{U}_{n}\right)\right\|_{L^{2}\left(\Omega \times Q_{t}\right)}^{2}+\frac{1}{2} E\left[\left\|\nabla\left(u_{n}-\hat{u}_{n}\right)(t)\right\|^{2}\right] \\
\leqslant & \frac{1}{2} E\left[\left\|\nabla\left(u_{0}-\hat{u}_{0}\right)\right\|^{2}\right]+\left\|\nabla\left(h_{n}-\hat{h}_{n}\right)\right\|_{L^{2}\left(\Omega \times Q_{t}\right)}^{2}+\frac{1}{2}\|g-\hat{g}\|_{L^{2}\left(\Omega \times Q_{t}\right)}^{2},
\end{aligned}
$$


and

$$
\begin{aligned}
& E\left[\left\|\left(U_{n}-\hat{U}_{n}\right)(t)\right\|^{2}\right] \\
& \leqslant e^{t}\left(\frac{2 C_{\alpha}^{2}}{\tilde{C}_{\alpha}+\frac{1}{2}}+T+4\right)\left\{\frac{1}{2}\left\|\nabla\left(u_{0}-\hat{u}_{0}\right)\right\|^{2}+\left\|\nabla\left(h_{n}-\hat{h}_{n}\right)\right\|_{L^{2}\left(\Omega \times Q_{t}\right)}^{2}\right. \\
&\left.+\frac{1}{2}\|g-\hat{g}\|_{L^{2}\left(\Omega \times Q_{t}\right)}^{2}\right\}+e^{t}\left\|u_{0}-\hat{u}_{0}\right\|^{2} .
\end{aligned}
$$

By adding (2.22) and (2.23) and passing to the limit one gets

$$
\begin{gathered}
\left(\tilde{C}_{\alpha}+\frac{1}{2}\right)\left\|\partial_{t}(U-\hat{U})\right\|_{L^{2}\left(\Omega \times Q_{t}\right)}^{2}+E\left[\|(U-\hat{U})(t)\|^{2}\right]+\frac{1}{2} E\left[\|\nabla(u-\hat{u})(t)\|^{2}\right] \\
\leqslant\left(1+e^{t}\left(\frac{2 C_{\alpha}^{2}}{\tilde{C}_{\alpha}+\frac{1}{2}}+T+4\right)\right)\left\{\frac{1}{2}\left\|\nabla\left(u_{0}-\hat{u}_{0}\right)\right\|^{2}+\|\nabla(h-\hat{h})\|_{L^{2}\left(\Omega \times Q_{t}\right)}^{2}\right. \\
\left.+\frac{1}{2}\|g-\hat{g}\|_{L^{2}\left(\Omega \times Q_{t}\right)}^{2}\right\}+e^{t}\left\|u_{0}-\hat{u}_{0}\right\|^{2}
\end{gathered}
$$

and since

$$
E\left[\|(u-\hat{u})(t)\|^{2}\right] \leqslant E\left[\|(U-\hat{U})(t)\|^{2}\right]+\|h-\hat{h}\|_{L^{2}\left(\Omega \times Q_{t}\right)}^{2},
$$

the announced result holds.

\section{Study of the multiplicative case with a nonlinear source term}

Under Assumptions $H_{2}$ to $H_{7}$, we are interested in the following problem with a multiplicative noise and a nonlinear source term:

$$
\left\{\begin{aligned}
\tilde{\alpha}\left(\partial_{t}\left(u-\int_{0} \mathscr{H}(u) d w\right)\right)-\Delta u+\beta(u) & =\vartheta \text { in }(0, T) \times D \times \Omega, \\
\nabla u . \mathbf{n} & =0 \text { on }(0, T) \times \partial D \times \Omega, \\
u(0, .) & =u_{0} .
\end{aligned}\right.
$$

Using Theorem 1.4, we define the application

$$
\begin{aligned}
\mathcal{T}: \mathcal{N}_{w}^{2}\left(0, T, H^{1}(D)\right) & \rightarrow \mathcal{N}_{w}^{2}\left(0, T, H^{1}(D)\right) \\
S & \mapsto u_{S},
\end{aligned}
$$

where $u_{S}$ is the solution of the following additive problem

$$
\left\{\begin{aligned}
\tilde{\alpha}\left(\partial_{t}\left(u_{S}-\int_{0} \mathscr{H}(S) d w\right)\right)-\Delta u_{S}+\beta(S) & =\vartheta \text { in }(0, T) \times D \times \Omega, \\
\nabla u_{S} \cdot \mathbf{n} & =0 \text { on }(0, T) \times \partial D \times \Omega, \\
u_{S}(0, .) & =u_{0}
\end{aligned}\right.
$$

in the sense of Definition 1.1 with $h=\mathscr{H}(S)$ and $g=\vartheta-\beta(S)$. Thus, for any $S, \hat{S}$ in $\mathcal{N}_{w}^{2}\left(0, T, H^{1}(D)\right)$, there exist $u_{S}=\mathcal{T}(S)$ and $u_{\hat{S}}=\mathcal{T}(\hat{S})$ such 
that by denoting $U_{S}=u_{S}-\int_{0} \mathscr{H}(S) d w$ and $U_{\hat{S}}=u_{\hat{S}}-\int_{0} \mathscr{H}(\hat{S}) d w$, we have using Proposition 1.5, for any $t \in[0, T]$ :

$$
\begin{aligned}
& \left(\tilde{C}_{\alpha}+\frac{1}{2}\right)\left\|\partial_{t}\left(U_{S}-U_{\hat{S}}\right)\right\|_{L^{2}\left(\Omega \times Q_{t}\right)}^{2}+E\left[\left\|\left(u_{S}-u_{\hat{S}}\right)(t)\right\|^{2}\right] \\
& +\frac{1}{2} E\left[\left\|\nabla\left(u_{S}-u_{\hat{S}}\right)(t)\right\|^{2}\right] \\
\leqslant & C_{\alpha}^{T}\left\{\|\nabla(\mathscr{H}(S)-\mathscr{H}(\hat{S}))\|_{L^{2}\left(\Omega \times Q_{t}\right)}^{2}+\frac{1}{2}\|\beta(S)-\beta(\hat{S})\|_{L^{2}\left(\Omega \times Q_{t}\right)}^{2}\right\} \\
& +\|\mathscr{H}(S)-\mathscr{H}(\hat{S})\|_{L^{2}\left(\Omega \times Q_{t}\right)}^{2} \\
\leqslant & K_{\alpha, \mathscr{H}}^{T}\left\{\|S-\hat{S}\|_{L^{2}\left(\Omega \times Q_{t}\right)}^{2}+\|\nabla(S-\hat{S})\|_{L^{2}\left(\Omega \times Q_{t}\right)}^{2}\right\},
\end{aligned}
$$

where

$$
K_{\alpha, \mathscr{H}}^{T}=C_{\alpha}^{T} \frac{C_{\beta}^{2}}{2}+\left(1+C_{\alpha}^{T}\right) C_{\mathscr{H}}^{2} \quad \text { and } \quad C_{\alpha}^{T}=1+e^{T}\left(\frac{2 C_{\alpha}^{2}}{\tilde{C}_{\alpha}+\frac{1}{2}}+T+4\right) .
$$

For any $a>0$ by using an integration by parts, one gets

$$
\begin{aligned}
& \int_{0}^{T} e^{-a t} E\left[\left\|\left(u_{S}-u_{\hat{S}}\right)(t)\right\|^{2}\right] d t+\int_{0}^{T} e^{-a t} E\left[\left\|\nabla\left(u_{S}-u_{\hat{S}}\right)(t)\right\|^{2}\right] d t \\
\leq & 2 K_{\alpha, \mathscr{H}}^{T} \int_{0}^{T} e^{-a t} \int_{0}^{t} E\left[\|(S-\hat{S})(s)\|^{2}+\|\nabla(S-\hat{S})(s)\|^{2}\right] d s d t \\
= & 2 K_{\alpha, \mathscr{H}}^{T} \times \frac{1}{a} \int_{0}^{T} e^{-a t} E\left[\|(S-\hat{S})(t)\|^{2}+\|\nabla(S-\hat{S})(t)\|^{2}\right] d t \\
& -2 K_{\alpha, \mathscr{H}}^{T} \times\left(\frac{1}{a} e^{-a T} \int_{0}^{T} E\left[\|(S-\hat{S})(t)\|^{2}+\|\nabla(S-\hat{S})(t)\|^{2}\right] d t\right) \\
\leq & 2 K_{\alpha, \mathscr{H}}^{T} \times \frac{1}{a} \int_{0}^{T} e^{-a t} E\left[\|(S-\hat{S})(t)\|^{2}+\|\nabla(S-\hat{S})(t)\|^{2}\right] d t .
\end{aligned}
$$

Finally

$$
\begin{aligned}
& \int_{0}^{T} e^{-a t} E\left[\|T(S)-T(\hat{S})\|_{H^{1}(D)}^{2}\right] d t \\
\leq & 2 K_{\alpha, \mathscr{H}}^{T} \times \frac{1}{a} \int_{0}^{T} e^{-a t} E\left[\|(S-\hat{S})(t)\|^{2}+\|\nabla(S-\hat{S})(t)\|^{2}\right] d t .
\end{aligned}
$$

Since the exponential weight in time provides in $\mathcal{N}_{w}^{2}\left(0, T, H^{1}(D)\right)$ an equivalent norm, under the condition $a>2 K_{\alpha, \mathscr{H}}^{T}, \mathcal{T}$ is a contractive mapping, it has a unique fixed-point and the result holds.

\section{Acknowledgment}

The authors wish to thank the joint program between the Higher Education Commission from Pakistan Ministry of Higher Education and the French Ministry of Foreign and European Affairs for the funding of A. Maitlo's PhD thesis. 


\section{References}

[1] S. N. Antontsev, G. Gagneux, R. Luce, and G. Vallet. New unilateral problems in stratigraphy. M2AN Math. Model. Numer. Anal., 40(4):765-784, 2006.

[2] S. N. Antontsev, G. Gagneux, R. Luce, and G. Vallet. A non-standard free boundary problem arising from stratigraphy. Anal. Appl. (Singap.), 4(3):209236, 2006.

[3] S. N. Antontsev, G. Gagneux, A. Mokrani, and G. Vallet. Stratigraphic modelling by the way of a pseudoparabolic problem with constraint. Adv. Math. Sci. Appl., 19(1):195-209, 2009.

[4] S. N. Antontsev, G. Gagneux, and G. Vallet. On some problems of stratigraphic control. Prikl. Mekh. Tekhn. Fiz., 44(6):85-94, 2003.

[5] Adimurthi, N. Seam, and G. Vallet. On the equation of Barenblatt-Sobolev. Commun. Contemp. Math., 13(5):843-862, 2011.

[6] G. I. Barenblatt. Scaling, self-similarity, and intermediate asymptotics, volume 14 of Cambridge Texts in Applied Mathematics. Cambridge University Press, Cambridge, 1996. With a foreword by Ya. B. Zeldovich.

[7] C. Bauzet, J. Giacomoni, and G. Vallet. On a class of quasilinear Barenblatt equations. Revista Real Academia de Ciencias de Zaragoza, 38:35-51, 2012.

[8] C. Bauzet and G. Vallet. On abstract Barenblatt equations. Differ. Equ. Appl., 3(4):487-502, 2011.

[9] G. Bonfanti, M. Frémond, and F. Luterotti. Global solution to a nonlinear system for irreversible phase changes. Advances in Mathematical Sciences and Applications, Gakkōtosho, Tokyo, Vol.10(1),1-23, 2000.

[10] H. Brezis. Analyse fonctionnelle. Collection Mathématiques Appliquées pour la Maîtrise. Masson, Paris, 1983. Théorie et applications.

[11] G. Da Prato and J. Zabczyk. Stochastic equations in infinite dimensions, volume 44 of Encyclopedia of Mathematics and its Applications. Cambridge University Press, Cambridge, 1992.

[12] J. Hulshof and J. L. Vázquez. Self-similar solutions of the second kind for the modified porous medium equation. European J. Appl. Math., 5(3):391-403, 1994.

[13] N. Igbida. Solutions auto-similaires pour une équation de Barenblatt. Rev. Mat. Apl., 17(1):21-36, 1996.

[14] S. Kamin, L. A. Peletier, and J. L. Vázquez. On the Barenblatt equation of elastoplastic filtration. Indiana Univ. Math. J., 40(4):1333-1362, 1991.

[15] J.-L. Lions. Quelques méthodes de résolution des problèmes aux limites non linéaires. Dunod, 1969.

[16] C. Prévôt and M. Röckner. A concise course on stochastic partial differential equations, volume 1905 of Lecture Notes in Mathematics. Springer, Berlin, 2007.

[17] M. Ptashnyk. Degenerate quaslinear pseudoparabolic equations with memory terms and variational inequalities. Nonlinear Anal., 66(12):2653-2675, 2007.

[18] J. Simon. Una generalización del teorema de Lions-Tartar. Bol. Soc. Esp. Mat. Apl., 40:43-69, 2007.

[19] G. Vallet. Sur une loi de conservation issue de la géologie. C. R. Math. Acad. Sci. Paris, 337(8):559-564, 2003. 
Caroline Bauzet

Aix-Marseille Université, CNRS, Centrale Marseille Laboratoire de Mécanique et d'Acoustique

4 impasse Nikola Tesla

13013 Marseille

France

e-mail: bauzet@lma.cnrs-mrs.fr

Frédéric Lebon

Aix-Marseille Université, CNRS, Centrale Marseille

Laboratoire de Mécanique et d'Acoustique

4 impasse Nikola Tesla

13013 Marseille

France

e-mail: lebon@lma.cnrs-mrs.fr

Asghar Maitlo

Aix-Marseille Université, CNRS, Centrale Marseille

Laboratoire de Mécanique et d'Acoustique

4 impasse Nikola Tesla

13013 Marseille

France

e-mail: maitlo@lma.cnrs-mrs.fr 